\title{
Some classical problems in random geometry
}

Pierre Calka

\begin{abstract}
This chapter is intended as a first introduction to selected topics in random geometry. It aims at showing how classical questions from recreational mathematics can lead to the modern theory of a mathematical domain at the interface of probability and geometry. Indeed, in each of the four sections, the starting point is a historical practical problem from geometric probability. We show that the solution of the problem, if any, and the underlying discussion are the gateway to the very rich and active domain of integral and stochastic geometry, which we describe at a basic level. In particular, we explain how to connect Buffon's needle problem to integral geometry, Bertrand's paradox to random tessellations, Sylvester's four-point problem to random polytopes and Jeffrey's bicycle wheel problem to random coverings. The results and proofs selected here have been especially chosen for non-specialist readers. They do not require much prerequisite knowledge on stochastic geometry but nevertheless comprise many of the main results on these models.
\end{abstract}

\section{Introduction: geometric probability, integral geometry, stochastic geometry}

Geometric probability is the study of geometric figures, usually from the Euclidean space, which have been randomly generated. The variables coming from these random spatial models can be classical objects from Euclidean geometry, such as a point, a line, a subspace, a ball, a convex polytope and so on.

It is commonly accepted that geometric probability was born in 1733 with Buffon's original investigation of the falling needle. Subsequently, several open questions appeared including Sylvester's four-point problem in 1864, Bertrand's paradox

Pierre Calka

University of Rouen, LMRS, avenue de l'université, BP 72, 76801 Saint-Étienne-du-Rouvray, email: pierre.calka@univ-rouen.fr 
related to a random chord in the circle in 1888 and Jeffreys's bicycle wheel problem in 1946. Until the beginning of the twentieth century, these questions were all considered as recreational mathematics and there was a very thin theoretical background involved which may explain why the several answers to Bertrand's question were regarded as a paradox.

After a course by G. Herglotz in 1933, W. Blaschke developed a new domain called integral geometry in his papers Integralgeometrie in 1935-1937, see e.g. [17]. It relies on the key idea that the mathematically natural probability models are those that are invariant under certain transformation groups and it provides mainly formulas for calculating expected values, i.e. integrals with respect to rotations or translations of random objects. Simultaneously, the modern theory of probability based on measure theory and Lebesgue's integral was introduced by S. N. Kolmogorov in [74].

During and after the Second World War, people with an interest in applications in experimental science - material physics, geology, telecommunications, etc.- realized the significance of random spatial models. For instance, in the famous foreword to the first edition of the reference book [31], D. G. Kendall narrates his own experience during the War and how his Superintendent asked him about the strength of a sheet of paper. This question was in fact equivalent to the study of a random set of lines in the plane. Similarly, J. L. Meijering published a first work on the study of crystal aggregates with random tessellations while he was working for the Philips company in 1953 [84]. In the same way, C. Palm who was working on telecommunications at Ericsson Technics proved a fundamental result in the one-dimensional case about what is nowadays called the Palm measure associated with a stationary point process [96]. All of these examples illustrate the general need to rigorously define and study random spatial models.

We traditionally consider that the expression stochastic geometry dates back to 1969 and was due to D. G. Kendall and K. Krickeberg at the occasion of the first conference devoted to that topic in Oberwolfach. In fact, I. Molchanov and W. S. Kendall note in the preface of [135] that H. L. Frisch and J. M. Hammersley had already written the following lines in 1963 in a paper on percolation: Nearly all extant percolation theory deals with regular interconnecting structures, for lack of knowledge of how to define randomly irregular structures. Adventurous readers may care to rectify this deficiency by pioneering branches of mathematics that might be called stochastic geometry or statistical topology.

For more than 50 years, a theory of stochastic geometry has been built in conjunction with several domains, including

- the theory of point processes and queuing theory, see notably the work of $\mathrm{J}$. Mecke [81], D. Stoyan [124], J. Neveu [95], D. Daley [35] and [42],

- convex and integral geometry, see e.g. the work of R. Schneider [112] and W. Weil [129] as well as their common reference book [114],

- the theory of random sets, mathematical morphology and image analysis, see the work of D. G. Kendall [69], G. Matheron [80] and J. Serra [117],

- combinatorial geometry, see the work of R. V. Ambartzumian [3]. 
It is worth noting that this development has been simultaneous with the research on spatial statistics and analysis of real spatial data coming from experimental science, for instance the work of B. Matérn in forestry [79] or the numerous papers in geostatistics, see e.g. [134].

In this introductory lecture, our aim is to describe some of the best-known historical problems in geometric probability and explain how solving these problems and their numerous extensions has induced a whole branch of the modern theory of stochastic geometry. We have chosen to embrace the collection of questions and results presented in this lecture under the general denomination of random geometry. In Section 1, Buffon's needle problem is used to introduce a few basic formulas from integral geometry. Section 2 contains a discussion around Bertrand's paradox which leads us to the construction of random lines and the first results on selected models of random tessellations. In Section 3, we present some partial answers to Sylvester's four-point problem and then derive from it the classical models of random polytopes. Finally, in Section 4, Jeffrey's bicycle wheel problem is solved and is the front door to more general random covering and continuum percolation.

We have made the choice to keep the discussion as non-technical as possible and to concentrate on the basic results and detailed proofs which do not require much prerequisite knowledge on the classical tools used in stochastic geometry. Each topic is illustrated by simulations which are done using Scilab 5.5. This chapter is intended as a foretaste of some of the topics currently most active in stochastic geometry and naturally encourages the reader to go beyond it and carry on learning with reference to books such as $[31,114,135]$.

Notation and convention. The Euclidean space $\mathbb{R}^{d}$ of dimension $d \geq 1$ and with origin denoted by $o$ is endowed with the standard scalar product $\langle\cdot, \cdot\rangle$, the Euclidean norm $\|\cdot\|$ and the Lebesgue measure $V_{d}$. The set $B_{r}(x)$ is the Euclidean ball centered at $x \in \mathbb{R}^{d}$ and of radius $r>0$. We denote by $\mathbb{B}^{d}$ (resp. $\mathbb{S}^{d-1}, \mathbb{S}_{+}^{d-1}$ ) the unit ball (resp. the unit sphere, the unit upper half-sphere). The Lebesgue measure on $\mathbb{S}^{d-1}$ will be denoted by $\sigma_{d}$. We will use the constant $\kappa_{d}=V_{d}\left(\mathbb{B}^{d}\right)=\frac{1}{d} \sigma_{d}\left(\mathbb{S}^{d-1}\right)=\frac{\pi^{\frac{d}{2}}}{\Gamma\left(\frac{d}{2}+1\right)}$. Finally, a convex compact set of $\mathbb{R}^{d}$ (resp. a compact intersection of a finite number of closed half-spaces of $\mathbb{R}^{d}$ ) will be called a $d$-dimensional convex body (resp. convex polytope).

\section{From Buffon's needle to integral geometry}

In this section, we describe and solve the four century-old needle problem due to Buffon and which is commonly considered as the very first problem in geometric probability. We then show how the solution to Buffon's original problem and to one of its extensions constitutes a premise to the modern theory of integral geometry. In particular, the notion of intrinsic volumes is introduced and two classical integral formulas involving them are discussed. 


\subsection{Starting from Buffon's needle}

In 1733, Georges-Louis Leclerc, comte de Buffon, raised a question which is nowadays better known as Buffon's needle problem. The solution, published in 1777 [20], is certainly a good candidate for the first-ever use of an integral calculation in probability theory. First and foremost, its popularity since then comes from being the first random experiment which provides an approximation of $\pi$.

Buffon's needle problem can be described in modern words in the following way: a needle is dropped at random onto a parquet floor which is made of parallel strips of wood, each of same width. What is the probability that it falls across a vertical line between two strips?

Let us denote by $D$ the width of each strip and by $\ell$ the length of the needle. We assume for the time being that $\ell \leq D$, i.e. that only one crossing is possible. The randomness of the experiment is described by a couple of real random variables, namely the distance $R$ from the needle's mid-point to the closest vertical line and the angle $\Theta$ between a horizontal line and the needle.

The chosen probabilistic model corresponds to our intuition of a random drop: the variables $R$ and $\Theta$ are assumed to be independent and both uniformly distributed on $(0, D / 2)$ and $(-\pi / 2, \pi / 2)$ respectively.

Now there is intersection if and only if $2 R \leq \ell \cos (\Theta)$. Consequently, we get

$$
p=\frac{2}{\pi D} \int_{-\frac{\pi}{2}}^{\frac{\pi}{2}} \int_{0}^{\frac{1}{2} \ell \cos (\theta)} \mathrm{d} r \mathrm{~d} \theta=\frac{2 \ell}{\pi D} .
$$

This remarkable identity leads to a numerical method for calculating an approximate value of $\pi$. Indeed, repeating the experiment $n$ times and denoting by $S_{n}$ the number of hits, we can apply Kolmogorov's law of large numbers to show that $\frac{2 \ell n}{D S_{n}}$ converges almost surely to $\pi$ with an error estimate provided by the classical central limit theorem.

In 1860, Joseph-Émile Barbier provided an alternative solution for Buffon's needle problem, see [6] and [73, Chapter 1]. We describe it below as it solves at the same time the so-called Buffon's noodle problem, i.e. the extension of Buffon's needle problem when the needle is replaced by any planar curve of class $C^{1}$.

Let us denote by $p_{k}, k \geq 0$, the probability of exactly $k$ crossings between the vertical lines and the needle. Henceforth, the condition $\ell \leq D$ is not assumed to be fulfilled any longer as it would imply trivially that $p=p_{1}$ and $p_{k}=0$ for every $k \geq 2$. We denote by $f(\ell)=\sum_{k \geq 1} k p_{k}$ the mean number of crossings. The function $f$ has the interesting property of being additive, i.e. if two needles of respective lengths $\ell_{1}$ and $\ell_{2}$ are pasted together at one of their endpoints and in the same direction, then the total number of crossing is obviously the sum of the numbers of crossings of the first needle and of the second one. This means that $f\left(\ell_{1}+\ell_{2}\right)=f\left(\ell_{1}\right)+f\left(\ell_{2}\right)$. Since the function $f$ is increasing, we deduce from its additivity that there exists a positive constant $\alpha$ such that $f(\ell)=\alpha \ell$.

More remarkably, the additivity property still holds when the two needles are not in the same direction. This implies that for any finite polygonal line $\mathscr{C}$, the mean 


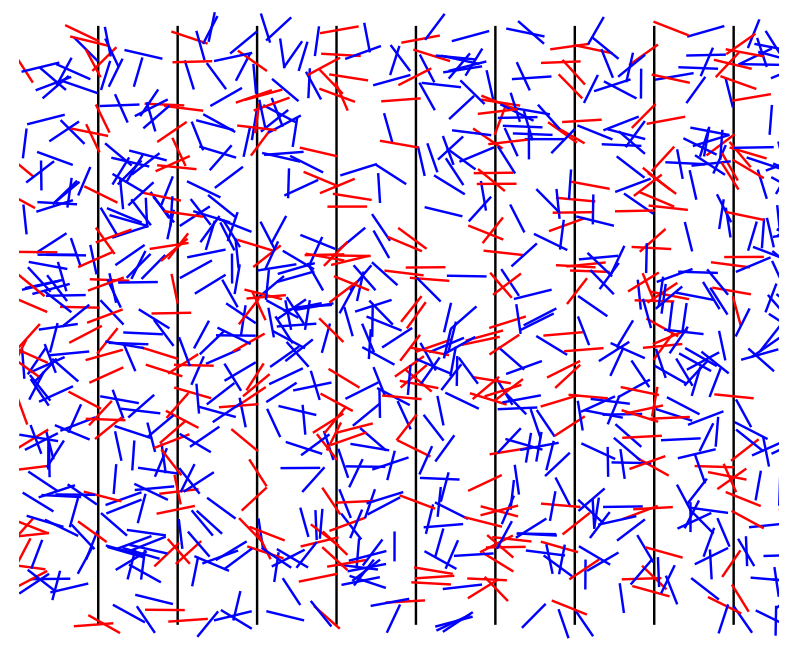

Fig. 1 Simulation of Buffon's needle problem with the particular choice $\ell / D=1 / 2$ : over 1000 samples, 316 were successful (red), 684 were not (blue).

number of crossings with the vertical lines of a rigid noodle with same shape as $\mathscr{C}$, denoted by $f(\mathscr{C})$ with a slight abuse of notation, satisfies

$$
f(\mathscr{C})=\alpha \mathscr{L}(\mathscr{C})
$$

where $\mathscr{L}(\cdot)$ denotes the arc length. Using both the density of polygonal lines in the space of piecewise $C^{1}$ planar curves endowed with the topology of uniform convergence and the continuity of the functions $f$ and $\mathscr{L}$ on this space, we deduce that the formula (1) holds for any piecewise $C^{1}$ planar curve.

It remains to make the constant $\alpha$ explicit, which we do when replacing $\mathscr{C}$ by the circle of diameter $D$. Indeed, almost surely, the number of crossings of this noodle with the vertical lines is 2 , which shows that $\alpha=\frac{2}{\pi D}$. In particular, when $K$ is a convex body of $\mathbb{R}^{2}$ with diameter less than $D$ and $p(K)$ denotes the probability that $K$ intersects one of the vertical lines, we get that

$$
p(K)=\frac{1}{2} f(\partial K)=\frac{\mathscr{L}(\partial K)}{\pi D} .
$$

Further extensions of Buffon's needle problem with more general needles and lattices can be found in [18]. In the next subsection, we are going to show how to derive the classical Cauchy-Crofton's formula from similar ideas. 


\subsection{Cauchy-Crofton formula}

We do now the opposite of Buffon's experiment, that is we fix a noodle which has the shape of a convex body $K$ of $\mathbb{R}^{2}$ and let a random line fall onto the plane. We then count how many times in mean the line intersects $K$.

This new experiment requires to define what a random line is, which means introducing a measure on the set of all lines of $\mathbb{R}^{2}$. We do so by using the polar equation of such a line, i.e. for any $\rho \in \mathbb{R}$ and $\theta \in[0, \pi)$, we denote by $L_{\rho, \theta}$ the line

$$
L_{\rho, \theta}=\rho(\cos (\theta), \sin (\theta))+\mathbb{R}(-\sin (\theta), \cos (\theta)) .
$$

Noticing that there is no natural way of constructing a probability measure on the set of random lines which would satisfy translation and rotation invariance, we endow the set $\mathbb{R} \times[0, \pi)$ with its Lebesgue measure. The integrated number of crossings of a line with a $C^{1}$ planar curve $\mathscr{C}$ is then represented by the function

$$
g(\mathscr{C})=\int_{-\infty}^{\infty} \int_{0}^{\pi} \#\left(L_{\rho, \theta} \cap \mathscr{C}\right) \mathrm{d} \theta \mathrm{d} \rho .
$$

The function $g$ is again additive under any concatenation of two curves so it is proportional to the arc length. A direct calculation when $\mathscr{C}$ is the unit circle then shows that

$$
g(\mathscr{C})=2 \mathscr{L}(\mathscr{C})
$$

This result is classically known in the literature as the Cauchy-Crofton formula [28, 34]. Going back to the initial question related to a convex body $K$, we apply (2) to $\mathscr{C}=\partial K$ and notice that $\#\left(L_{\rho, \theta} \cap \mathscr{C}\right)$ is equal to $2 \mathbf{1}_{\left\{L_{\rho, \theta} \cap K \neq \emptyset\right\}}$. We deduce that

$$
\mathscr{L}(\partial K)=\int_{-\infty}^{\infty} \int_{0}^{\pi} \mathbf{1}_{\left\{L_{\rho, \theta} \cap K \neq \emptyset\right\}} \mathrm{d} \theta \mathrm{d} \rho .
$$

\subsection{Extension to higher dimension}

We aim now at extending (3) to higher dimension, that is we consider the set $\mathscr{K}^{d}$ of convex bodies of $\mathbb{R}^{d}, d \geq 2$, and for any element $K$ of $\mathscr{K}^{d}$, we plan to calculate integrals over all possible $k$-dimensional affine subspaces $L_{k}$ of the content of $L_{k} \cap K$. This requires to introduce a set of fundamental functionals called intrinsic volumes on the space of convex bodies of $\mathbb{R}^{d}$. This is done through the rewriting of the volume of the parallel set $\left(K+B_{\rho}(o)\right)$ as a polynomial in $\rho>0$. Indeed, we are going to prove that there exists a unique set of $d$ functions $V_{0}, \cdots, V_{d-1}$ such that

$$
V_{d}\left(K+B_{\rho}(o)\right)=\sum_{k=0}^{d} \kappa_{d-k} \rho^{d-k} V_{k}(K) \text {. }
$$


The identity (4) is known under the name of Steiner formula and was proved by Steiner for $d=2$ and 3 in 1840 [122]. In particular, the renormalization with the multiplicative constant $\kappa_{d-k}$ guarantees that the quantity $V_{k}(K)$ is really intrinsic to $K$, i.e. that it does not depend on the dimension of the underlying space. We explain below how to prove (4), following closely [113, Section 1].

In the first step, we start by treating the case when $K$ is a convex polytope $P$. We denote by $\mathscr{F}$ the set of all faces of $P$ and by $\mathscr{F}_{k}, 0 \leq k \leq d$, the subset of $\mathscr{F}$ consisting of the $k$-dimensional faces of $P$. For any face $F \in \mathscr{F} \backslash\{P\}$, the open outer normal cone of $F$ denoted by $N_{P}(F)$ is the set of $x \in \mathbb{R}^{d} \backslash\{o\}$ such that there exists an affine hyperplane $H$ with normal vector $x$ satisfying $H \cap P=F$ and $\langle x, y-h\rangle \leq 0$ for every $y \in P$ and $h \in H$. In particular, when $F \in \mathscr{F}_{k}, 0 \leq k \leq(d-1), N_{P}(F)$ is a $(d-k)$-dimensional cone. Moreover, $\left(F+N_{P}(F)\right)$ is the set of points outside $P$ whose nearest point in $P$ lies in $F$ and no other lower-dimensional face. The set of all sets $\left(F+N_{P}(F)\right)$, often called the normal fan of $P$, is a partition of $\mathbb{R}^{d} \backslash P$, see Figure 2. Consequently, from the decomposition of $\left(P+B_{\rho}(o)\right) \backslash P$ combined with Fubini's theorem, we get

$$
\begin{aligned}
V_{d}\left(P+B_{\rho}(o)\right) & =V_{d}(P)+\sum_{F \in \mathscr{F} \backslash\{P\}} V_{d}\left(F+\left(N_{P}(F) \cap B_{\rho}(o)\right)\right) \\
& =V_{d}(P)+\sum_{k=0}^{d-1} \sum_{F \in \mathscr{F}_{k}} V_{k}(F) \gamma(F, P) \rho^{d-k} \kappa_{d-k}
\end{aligned}
$$

where $\gamma(F, P)$ is the normalized area measure on the unit sphere $\mathbb{S}^{d-k-1}$ of $N_{P}(F) \cap$ $\mathbb{S}^{d-k-1}$. In particular, we deduce from (5) that (4) holds for $K=P$ as soon as

$$
V_{k}(P)=\sum_{F \in \mathscr{F}_{k}} V_{k}(F) \gamma(F, P) .
$$

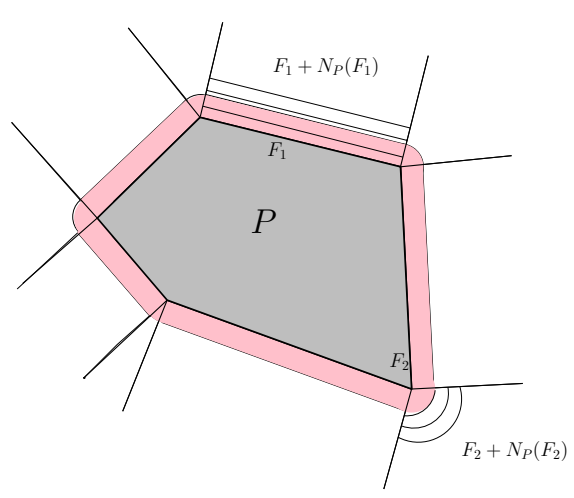

Fig. 2 A two-dimensional convex polygon $P$ (gray), the region $\left(P+B_{r}(o)\right) \backslash P$ (pink), the sets $\left(F+N_{P}(F)\right)$ with two examples for the edge $F_{1}$ and the vertex $F_{2}$ (striped regions). 
In the second step, we derive the Steiner formula for any convex body $K$. In order to define $V_{k}(K)$, we use the trick to rewrite (4) for a polytope $P$ and for several values of $\rho$, namely $\rho=1, \cdots,(d+1)$. The $(d+1)$ equalities constitute a Vandermonde system of $(d+1)$ equations in $\left(\kappa_{d} V_{0}(P), \cdots, \kappa_{0} V_{d}(P)\right)$. These equations are linearly independent because the Vandermonde determinant of $n$ pairwise distinct real numbers is different from zero. When solving the system by inverting the Vandermonde matrix, we construct a sequence $\alpha_{k, l}, 0 \leq k \leq d, 1 \leq l \leq(d+1)$ such that for every polytope $P$ and $0 \leq k \leq d$,

$$
V_{k}(P)=\sum_{l=1}^{d+1} \alpha_{k, l} V_{d}\left(P+B_{l}(o)\right) .
$$

It remains to check that the set of functions $V_{k}(\cdot)=\sum_{l=1}^{d+1} \alpha_{k, l} V_{d}\left(\cdot+B_{l}(o)\right), 0 \leq k \leq$ $d$, defined on $\mathscr{K}^{d}$ satisfies (4). This follows from the continuity of $V_{d}$, and hence of all $V_{k}$ on the space $\mathscr{K}^{d}$ endowed with the Hausdorff metric and from the fact that (4) holds on the set of convex polytopes which is dense in $\mathscr{K}^{d}$.

For practical reasons, we extend the definition of intrinsic volumes to $K=\emptyset$ by taking $V_{k}(\emptyset)=0$ for every $k \geq 0$. Of particular interest are:

- the functional $V_{0}$ equal to $\mathbf{1}_{\{K \neq \emptyset\}}$,

- the functional $V_{1}$ equal to the so-called mean width up to the multiplicative constant $d \kappa_{d} /\left(2 \kappa_{d-1}\right)$,

- the functional $V_{d-1}$ equal to half of the Hausdorff measure of $\partial K$.

Furthermore, Hadwiger's theorem, which asserts that any additive, continuous and motion-invariant function on $\mathscr{K}^{d}$ is a linear combination of the $V_{k}$ 's, provides an alternative way of characterizing intrinsic volumes [51].

We now go back to our initial purpose, i.e. extending the Cauchy-Crofton formula to higher dimension. We do so in two different ways:

First, when $K \in \mathscr{K}^{2}$, an integration over $\rho$ in (3) shows that

$$
\mathscr{L}(\partial K)=2 V_{1}(K)=\int_{0}^{\pi} V_{1}\left(K \mid L_{0, \theta}\right) \mathrm{d} \theta
$$

where $\left(K \mid L_{0, \theta}\right)$ is the one-dimensional orthogonal projection of $K$ onto $L_{0, \theta}$. When $K \in \mathscr{K}^{d}, d \geq 2$, Kubota's formula asserts that any intrinsic volume $V_{k}$ can be recovered up to a multiplicative constant as the mean of the Lebesgue measure of the projection of $K$ onto a uniformly distributed random $k$-dimensional linear subspace. In other words, there exists an explicit positive constant $c$ depending on $d$ and $k$ but not on $K$ such that for every $K \in \mathscr{K}^{d}$,

$$
V_{k}(K)=c \int_{\mathrm{SO}_{d}} V_{k}(K \mid \mathscr{R}(L)) \mathrm{d} v_{d}(\mathscr{R})
$$

where $L$ is a fixed $k$-dimensional linear subspace of $\mathbb{R}^{d}, \mathrm{SO}_{d}$ is the usual special orthogonal group of $\mathbb{R}^{d}$ and $v_{d}$ is its associated normalized Haar measure.

Secondly, with a slight rewriting of (3), we get for any $K \in \mathscr{K}^{2}$ 


$$
\mathscr{L}(\partial K)=\iint_{\mathbb{R} \times(0, \pi)} V_{o}\left(K \cap\left(\operatorname{rot}_{\theta}\left(L_{0,0}\right)+\rho(\cos (\theta), \sin (\theta))\right)\right) \mathrm{d} \rho \mathrm{d} \theta,
$$

where $\operatorname{rot}_{\theta}$ is the rotation around $o$ and of angle $\theta$. When $K \in \mathscr{K}^{d}, d \geq 2$, for any $0 \leq l \leq k \leq d$ and any fixed $(d-k+l)$-dimensional linear subspace $L_{d-k+l}$, the Crofton formula states that the $k$-th intrinsic volume of $K$ is proportional to the mean of the $l$-th intrinsic volume of the intersection of $K$ with a uniformly distributed random $(d-k+l)$-dimensional affine subspace, i.e. there exists an explicit positive constant $c^{\prime}$ depending on $d, k$ and $l$ but not on $K$ such that

$$
V_{k}(K)=c^{\prime} \int_{\mathrm{SO}_{d}} \int_{L_{d-k+l}^{\perp}} V_{l}\left(K \cap\left(\mathscr{R} L_{d-k+l}+t\right)\right) \mathrm{d} t \mathrm{~d} v_{d}(\mathscr{R}),
$$

where $L_{d-k+l}$ is a fixed $(d-k+l)$-dimensional affine subspace of $\mathbb{R}^{d}$.

For the proofs of (6) and (7) with the proper explicit constants and for a more extensive account on integral geometry and its links to stochastic geometry, we refer the reader to the reference books [111, Chapters 13-14], [110, Chapters 4-5] and [114, Chapters 5-6]. We will show in the next section how some of the formulas from integral geometry are essential to derive explicit probabilities related to the Poisson hyperplane tessellation.

\section{From Bertrand's paradox to random tessellations}

In this section, we recall Bertrand's problem which leads to three different and perfectly correct answers. This famous paradox questions the several potential models for constructing random lines in the Euclidean plane. The fundamental choice of the translation-invariance leads us to the definition of the stationary Poisson line process. After that, by extension, we survey a few basic facts on two examples of stationary random tessellations of $\mathbb{R}^{d}$, namely the Poisson hyperplane tessellation and the Poisson-Voronoi tessellation.

\subsection{Starting from Bertrand's paradox}

In the book entitled Calcul des Probabilités and published in 1889 [12], J. Bertrand asks for the following question: a chord of the unit circle is chosen at random. What is the probability that it is longer than $\sqrt{3}$, i.e. the edge of an equilateral triangle inscribed in the circle?

The paradox comes from the fact that there are several ways of choosing a chord at random. Depending on the model that is selected, the question has several possible answers. In particular, there are three correct calculations which show that the required probability is equal to either $1 / 2$, or $1 / 3$ or $1 / 4$. Still a celebrated and wellknown mathematical brain-teaser, Bertrand's problem questions the foundations of 
the modern probability theory when the considered variables are not discrete. We describe below the three different models and solutions.

Solution 1: random radius. We define a random chord through the polar coordinates $(R, \Theta)$ of the orthogonal projection of the origin onto it. The variable $\Theta$ is assumed to be uniformly distributed on $(0,2 \pi)$ because of the rotation-invariance of the problem while $R$ is taken independent of $\Theta$ and uniformly distributed in $(0,1)$. The length of the associated chord is $2 \sqrt{1-R^{2}}$. Consequently, the required probability is

$$
p_{1}=P\left(2 \sqrt{1-R^{2}} \geq \sqrt{3}\right)=P(R \leq 1 / 2)=1 / 2 .
$$

Solution 2: random endpoints. We define a random chord through the position $\Theta$ of its starting point in the anticlockwise direction and the circular length $\Theta^{\prime}$ to its endpoint. Again, $\Theta$ is uniformly distributed on $(0,2 \pi)$ while $\Theta^{\prime}$ is chosen independent of $\Theta$ and also uniformly distributed in $(0,2 \pi)$. The length of the associated chord is $2 \sin \left(\Theta^{\prime} / 2\right)$. Consequently, the required probability is

$$
p_{2}=P\left(2 \sin \left(\Theta^{\prime} / 2\right) \geq \sqrt{3}\right)=P\left(\Theta^{\prime} / 2 \in(\pi / 3,2 \pi / 3)\right)=\frac{\frac{4 \pi}{3}-\frac{2 \pi}{3}}{2 \pi}=\frac{1}{3} .
$$

Solution 3: random midpoint. We define a random chord through its midpoint $X$. The random point $X$ is assumed to be uniformly distributed in the unit disk. The length of the associated chord is $2 \sqrt{1-\|X\|^{2}}$. Consequently, the required probability is

$$
p_{3}=P(\|X\| \leq 1 / 2)=\frac{V_{2}\left(B_{o}(1 / 2)\right)}{V_{2}\left(B_{o}(1)\right)}=\frac{1}{4} .
$$
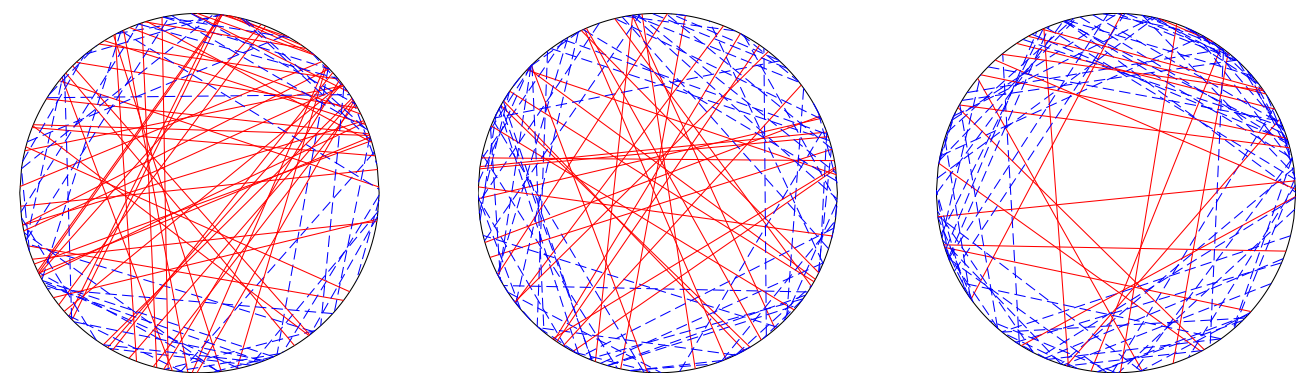

Fig. 3 Simulation of Bertrand's problem with 100 chords: (a) Solution 1 (left): 54 successful (plain line, red), 46 unsuccessful (dotted line, blue) (b) Solution 2 (middle): 30 successful (c) Solution 3 (right): 21 successful.

In conclusion, as soon as the model, i.e. the meaning that is given to the word random, is fixed, all three solutions look perfectly correct. J. Bertrand considers the problem as ill-posed, that is he does not decide in favor of any of the three. Neither does H. Poincaré in his treatment of Bertrand's paradox in his own Calcul des prob- 
abilités in 1912 [101]. Actually, they build on it a tentative formalized probability theory in a continuous space. Many years later, in his 1973 paper The well-posed problem [68], E. T. Jaynes explains that a natural way for discriminating between the three solutions consists in favoring the one which has the most invariance properties with respect to transformation groups. All three are rotation invariant but only one is translation invariant and that is Solution 1. And in fact, in a paper from 1868 [34], long before Bertrand's book, M. W. Crofton had already proposed a way to construct random lines which guarantees that the mean number of lines intersecting a fixed closed convex set is proportional to the arc length of its boundary.

Identifying the set of lines $L_{\rho, \theta}$ with $\mathbb{R} \times[0, \pi)$, we observe that the previous discussion means that the Lebesgue measure $\mathrm{d} \rho \mathrm{d} \theta$ on $\mathbb{R} \times[0, \pi)$ plays a special role when generating random lines. Actually, it is the only rotation and translation invariant measure up to a multiplicative constant. The construction of a natural random set of lines in $\mathbb{R}^{2}$ will rely heavily on it.

\subsection{Random sets of points, random sets of lines and extensions}

Generating random geometric shapes in the plane requires to generate random sets of points and random sets of lines. Under the restriction to a fixed convex body $K$, the most natural way to generate random points consists in constructing a sequence of independent points which are uniformly distributed in $K$. Similarly, in view of the conclusion on Bertrand's paradox, random lines can be naturally taken as independent and identically distributed lines with common distribution

$$
\frac{1}{\mu_{2}(K)} \mathbf{1}_{\left\{L_{\rho, \theta} \cap K \neq \emptyset\right\}} \mathrm{d} \rho \mathrm{d} \theta
$$

where

$$
\mu_{2}(\cdot)=\iint_{\mathbb{R} \times(0, \pi)} \mathbf{1}_{\left\{L_{\rho, \theta} \cap \cdot \neq \emptyset\right\}} \mathrm{d} \rho \mathrm{d} \theta .
$$

These constructions present two drawbacks: first, they are only defined inside $K$ and not in the whole space and secondly, they lead to undesired dependencies. Indeed, when fixing the total number of points or lines thrown in $K$, the joint distribution of the numbers of points or lines falling into several disjoint Borel subsets of $K$ is multinomial. Actually, there is a way of defining a more satisfying distribution on the space of locally finite sets of points (resp. lines) in the plane endowed with the $\sigma$-algebra generated by the set of functions which to any set of points (resp. lines) associates the number of points falling into a fixed Borel set of $\mathbb{R}^{2}$ (resp. the number of lines intersecting a fixed Borel set of $\mathbb{R}^{2}$ ). Indeed, for any fixed $\lambda>0$, there exists a random set of points (resp. lines) in the plane such that:

- for every Borel set $B$ with finite Lebesgue measure, the number of points falling into $B$ (resp. the number of lines intersecting $B$ ) is Poisson distributed with mean $\lambda V_{2}(B)\left(\right.$ resp. $\left.\lambda \mu_{2}(B)\right)$ 
- for every finite collection of disjoint Borel sets $B_{1}, \cdots, B_{k}, k \geq 1$, the numbers of points falling into $B_{i}$ (resp. lines intersecting $B_{i}$ ) are mutually independent.

This random set is unique in distribution and both translation and rotation invariant. It is called a homogeneous Poisson point process (resp. isotropic and stationary Poisson line process) of intensity $\lambda$. For a detailed construction of both processes, we refer the reader to e.g. [114, Section 3.2].

The Poisson point process can be naturally extended to $\mathbb{R}^{d}, d \geq 3$, by replacing $V_{2}$ with $V_{d}$. Similarly, we define the isotropic and stationary Poisson hyperplane process in $\mathbb{R}^{d}$ by replacing $\mu_{2}$ by a measure $\mu_{d}$ which is defined in the following way. For any $\rho \in \mathbb{R}$ and $\mathbf{u} \in \mathbb{S}_{+}^{d-1}$, we denote by $H_{\rho, \mathbf{u}}$ the hyperplane containing the point $\rho \mathbf{u}$ and orthogonal to $\mathbf{u}$. Let $\mu_{d}$ be the measure on $\mathbb{R}^{d}$ such that for any Borel set $B$ of $\mathbb{R}^{d}$,

$$
\mu_{d}(B)=\iint_{\mathbb{R} \times \mathbb{S}_{+}^{d-1}} \mathbf{1}_{\left\{H_{\rho, \mathbf{u}} \cap B \neq \emptyset\right\}} \mathrm{d} \rho \mathrm{d} \sigma_{d}(\mathbf{u}) .
$$

Another possible extension of these models consists in replacing the Lebesgue measure $V_{d}$ (resp. the measure $\mu_{d}$ ) by any locally finite measure which is not a multiple of $V_{d}$ (resp. of $\mu_{d}$ ). This automatically removes the translation invariance in the case of the Poisson point process while the translation invariance is preserved in the case of the Poisson hyperplane process only if the new measure is of the form $\mathrm{d} \rho \mathrm{d} v_{d}(\mathbf{u})$ where $v_{d}$ is a measure on $\mathbb{S}_{+}^{d-1}$. For more information on this and also on non-Poisson point processes, we refer the reader to the reference books [95, 36, $72,31]$. In what follows, we only consider homogeneous Poisson point processes and isotropic and stationary Poisson hyperplane processes, denoted respectively by $\mathscr{P}_{\lambda}$ and $\widehat{\mathscr{P}}_{\lambda}$. These processes will constitute the basis for constructing stationary random tessellations of the Euclidean space.

\subsection{On two examples of random convex tessellations}

The Poisson hyperplane process $\widehat{\mathscr{P}}_{\lambda}$ induces naturally a tessellation of $\mathbb{R}^{d}$ into convex polytopes called cells which are the closures of the connected components of the set $\mathbb{R}^{d} \backslash \bigcup_{H \in \widehat{\mathscr{P}_{\lambda}}} H$. This tessellation is called the (isotropic and stationary) Poisson hyperplane tessellation of intensity $\lambda$. In dimension two, with probability one, any crossing between two lines is an X-crossing, i.e. any vertex of a cell belongs to exactly 4 different cells while in dimension $d$, any $k$-dimensional face of a cell, $0 \leq k \leq d$, is included in the intersection of $(d-k)$ different hyperplanes and belongs to exactly $2^{d-k}$ different cells almost surely.

Similarly, the Poisson point process $\mathscr{P}_{\lambda}$ also generates a tessellation in the following way: any point $x$ of $\mathscr{P}_{\lambda}$, called a nucleus, gives birth to its associated cell $C\left(x \mid \mathscr{P}_{\lambda}\right)$ defined as the set of points of $\mathbb{R}^{d}$ which are closer to $x$ than to any other point of $\mathscr{P}_{\lambda}$ for the Euclidean distance, i.e. 


$$
C\left(x \mid \mathscr{P}_{\lambda}\right)=\left\{y \in \mathbb{R}^{d}:\|y-x\| \leq\left\|y-x^{\prime}\right\| \forall x^{\prime} \in \mathscr{P}_{\lambda}\right\} .
$$

This tessellation is called the Voronoi tessellation generated by $\mathscr{P}_{\lambda}$ or the PoissonVoronoi tessellation in short. In particular, the cell $C\left(x \mid \mathscr{P}_{\lambda}\right)$ with nucleus $x$ is bounded by portions of bisecting hyperplanes of segments $\left[x, x^{\prime}\right], x^{\prime} \in \mathscr{P}_{\lambda} \backslash\{x\}$. The set of vertices and edges of these cells constitute a graph which is random and embedded in $\mathbb{R}^{d}$, sometimes referred to as the Poisson-Voronoi skeleton. In dimension two, with probability one, any vertex of this graph belongs to exactly three different edges and three different cells while in dimension $d$, any $k$-dimensional face of a cell, $0 \leq k \leq d$, is included in the intersection of $(d+1-k)(d-k) / 2$ different bisecting hyperplanes and belongs to exactly $(d+1-k)$ different cells almost surely.
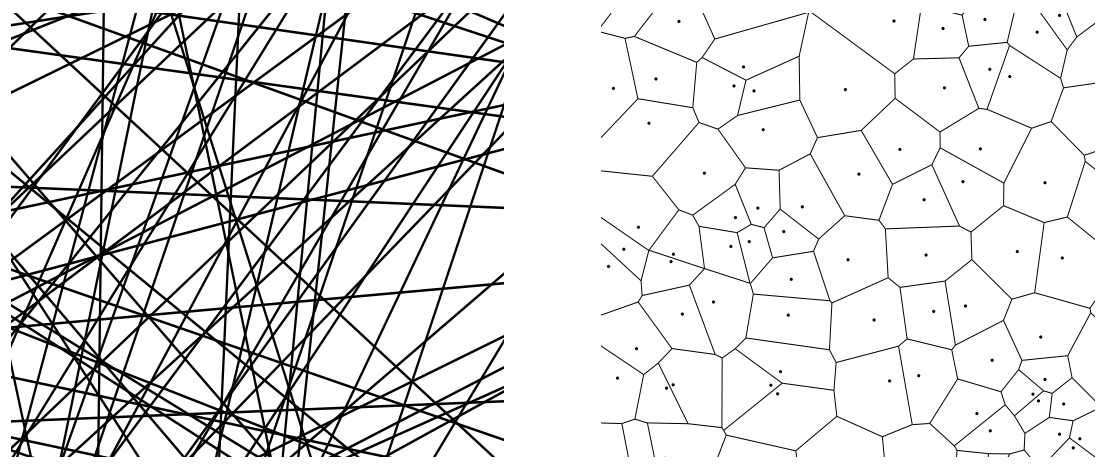

Fig. 4 Simulation of the Poisson line tessellation (left) and the Poisson-Voronoi tessellation (right) in the square.

The Poisson hyperplane tessellation has been used as a natural model for the trajectories of particles inside bubble chambers [46], the fibrous structure of paper [85] and the road map of a city [56]. The Voronoi construction was introduced in the first place by R. Descartes as a possible model for the shape of the galaxies in the Universe [37]. The Poisson-Voronoi tessellation has appeared since then in numerous applied domains, including telecommunication networks [5] and materials science $[103,76]$.

In both cases, stationarity of the underlying Poisson process makes it possible to do a statistical study of the tessellation. Indeed, let $f$ be a translation-invariant, measurable and non-negative real-valued function defined on the set $\mathscr{P}^{d}$ of convex polytopes of $\mathbb{R}^{d}$ endowed with the topology of the Hausdorff distance. For $r>0$, let $\mathbf{C}_{r}$ and $N_{r}$ be respectively the set of cells included in $B_{r}(o)$ and its cardinality. Then, following for instance [33], we can apply Wiener's ergodic theorem to get that, when $r \rightarrow \infty$, 


$$
\frac{1}{N_{r}} \sum_{C \in \mathbf{C}_{r}} f(C) \rightarrow \frac{1}{E\left(V_{d}\left(C_{o}\right)^{-1}\right)} E\left(\frac{f\left(C_{o}\right)}{V_{d}\left(C_{o}\right)}\right) \quad \text { almost surely }
$$

where $C_{o}$ is the almost-sure unique cell containing the origin $o$ in its interior.

This implies that two different cells are of particular interest: the cell $C_{o}$, often called the zero-cell, and the cell $\mathscr{C}$ defined in distribution by the identity

$$
E(f(\mathscr{C}))=\frac{1}{E\left(V_{d}\left(C_{o}\right)^{-1}\right)} E\left(\frac{f\left(C_{o}\right)}{V_{d}\left(C_{o}\right)}\right) .
$$

The convergence at (9) suggests that $\mathscr{C}$ has the law of a cell chosen uniformly at random in the whole tessellation, though such a procedure would not have any clear mathematical meaning. That is why $\mathscr{C}$ is called the typical cell of the tessellation even if it is not defined almost surely and it does not belong to the tessellation either. In particular, the typical cell is not equal in distribution to the zero-cell and is actually stochastically smaller since it has a density proportional to $V_{d}\left(C_{o}\right)^{-1}$ with respect to the distribution of $C_{o}$. Actually, it is possible in the case of the Poisson hyperplane tessellation to construct a realization of $\mathscr{C}$ which is almost surely strictly included in $C_{o}$ [82]. This fact can be reinterpreted as a multidimensional version of the classical bus waiting time paradox, which says the following: if an individual arrives at time $t$ at a bus stop, the time between the last bus he/she missed and the bus he/she will take is larger than the typical interarrival time between two consecutive busses.

Relying on either (9) or (10) may not be easy when calculating explicit mean values or distributions of geometric characteristics of $\mathscr{C}$. Another equivalent way of defining the distribution of $\mathscr{C}$ is provided by the use of a so-called Palm distribution, see e.g. [96], [92, Section 3.2], [114, Sections 3.3,3.4] and [75, Section 9]. For sake of simplicity, we explain the procedure in the case of the Poisson-Voronoi tessellation only. Let $f$ be a measurable and non-negative real-valued function defined on $\mathscr{P}^{d}$. Then, for any Borel set $B$ such that $0<V_{d}(B)<\infty$, we get

$$
E(f(\mathscr{C}))=\frac{1}{\lambda V_{d}(B)} E\left(\sum_{x \in \mathscr{P}_{\lambda} \cap B} f\left(C\left(x \mid \mathscr{P}_{\lambda}\right)-x\right)\right) .
$$

where $C\left(x \mid \mathscr{P}_{\lambda}\right)-x$ is the set $C\left(x \mid \mathscr{P}_{\lambda}\right)$ translated by $-x$. The fact that the quantity on the right-hand side of the identity (11) does not depend on the Borel set $B$ comes from the translation invariance of the Poisson point process $\mathscr{P}_{\lambda}$. It is also remarkable that the right-hand side of (11) is again a mean over the cells with nucleus in $B$ but contrary to (9) there is no limit involved, which means in particular that $B$ can be as small as one likes, provided that $V_{d}(B)>0$.

Following for instance [92, Proposition 3.3.2], we describe below the proof of (11), i.e. that for any translation-invariant, measurable and non-negative function $f$, the Palm distribution defined at (11) is the same as the limit of the means in the law of large numbers at (9). Indeed, let us assume that $\mathscr{C}$ be defined in law by the identity (11) and let us prove that (10) is satisfied, i.e. that $\mathscr{C}$ has a density 
proportional to $V_{d}(\cdot)^{-1}$ with respect to $C_{o}$. By classical arguments from measure theory, (11) implies that for any non-negative measurable function $F$ defined on the product space $\mathscr{P}^{d} \times \mathbb{R}^{d}$,

$$
\lambda \int E(F(\mathscr{C}, x)) \mathrm{d} x=E\left(\sum_{x \in \mathscr{P}_{\lambda}} F\left(C\left(x \mid \mathscr{P}_{\lambda}\right)-x, x\right)\right) .
$$

Applying this to $F(C, x)=\frac{f(C)}{V_{d}(C)} \mathbf{1}_{\{-x \in C\}}$ and using the translation invariance of $f$, we get

$$
\lambda E(f(\mathscr{C}))=E\left(\sum_{x \in \mathscr{P}_{\lambda}} \frac{f\left(C\left(x \mid \mathscr{P}_{\lambda}\right)\right)}{V_{d}\left(C\left(x \mid \mathscr{P}_{\lambda}\right)\right)} \mathbf{1}_{o \in C\left(x \mid \mathscr{P}_{\lambda}\right)}\right)=E\left(\frac{f\left(C_{o}\right)}{V_{d}\left(C_{o}\right)}\right) .
$$

Applying (12) to $f=1$ and $f=V_{d}$ successively, we get

$$
E\left(V_{d}(\mathscr{C})\right)=\frac{1}{E\left(V_{d}\left(C_{o}\right)^{-1}\right)}=\frac{1}{\lambda} .
$$

Combining (12) and (13), we obtain that the typical cell $\mathscr{C}$ defined at (11) satisfies (10) so it is equal in distribution to the typical cell defined earlier through the law of large numbers at (9).

In addition to these two characterizations of the typical cell, the Palm definition (11) of $\mathscr{C}$ in the case of the Poisson-Voronoi tessellation provides a very simple realization of the typical cell $\mathscr{C}:$ it is equal in distribution to the cell $C\left(o \mid \mathscr{P}_{\lambda} \cup\{o\}\right)$, i.e. the Voronoi cell associated with the nucleus $o$ when the origin is added to the set of nuclei of the tessellation. This result is often called Slivnyak's theorem, see e.g. [114, Theorem 3.3.5].

The classical problems related to stationary tessellations are mainly the following:

(a) making a global study of the tessellation, for instance on the set of vertices or edges: calculation of mean global topological characteristics per unit volume, proof of limit theorems, etc;

(b)calculating mean values and whenever possible, moments and distributions of geometric characteristics of the zero-cell or the typical cell or a typical face;

(c) studying rare events, i.e. estimating distribution tails of the characteristics of the zero-cell or the typical cell and proving the existence of limit shapes in some asymptotic context.

Problem (a). As mentioned earlier, R. Cowan [33] showed several laws of large numbers by ergodic methods which were followed by second-order results from the seminal work due to F. Avram and Bertsimas on central limit theorems [4] to the more recent additions [55] and [57]. Topological relationships have been recently established in [130] for a general class of stationary tessellations. 
Problem (b). The question of determining mean values of the volume or any combinatorial quantity of a particular cell was tackled early. The main contributions are due notably to G. Matheron [80, Chapter 6] and R. E. Miles [87, 88] in the case of the Poisson hyperplane tessellation and to J. Møller [90] in the case of the Poisson-Voronoi tessellation. Still, to the best of our knowledge, some mean values are unknown like for instance the mean number of $k$-dimensional faces of the Poisson-Voronoi typical cell for $1 \leq k \leq(d-1)$ and $d \geq 3$. Regarding explicit distributions, several works are noticeable $[11,22,23]$ but in the end, it seems that very few have been computable up to now.

Problem (c). The most significant works related to this topic have been rather recent: distribution tails and large-deviations type results [44, 58], extreme values [29], high dimension [59]... Nevertheless, many questions, for instance regarding precise estimates of distribution tails, remain open to this day. One of the most celebrated questions concerns the shape of large cells. A famous conjecture stated by D. G. Kendall in the forties asserts that large cells from a stationary and isotropic Poisson line tessellation are close to the circular shape, see e.g. the foreword to [31]. This remarkable feature is in fact common to the Crofton cell and the typical cell of both the Poisson hyperplane tessellation and the Poisson-Voronoi tessellation in any dimension. It was formalized for different meanings of large cells and proved, with an explicit probability estimate of the deviation to the limit shape, by D. Hug, M. Reitzner and R. Schneider in a series of breakthrough papers, see e.g. [63, 64, 60].

Intentionally, we have chosen to skip numerous other models of tessellations. Noteworthy among these are those generated by non-Poisson point processes [45], Johnson-Mehl tessellations [91] and especially STIT tessellations [94].

In the next subsection, we collect a few explicit first results related to Problem (b), i.e. the mean value and distribution of several geometric characteristics of either the zero-cell $C_{o}$ or the typical cell $\mathscr{C}$.

\subsection{Mean values and distributional properties of the zero-cell and of the typical cell}

This subsection is not designed as an exhaustive account on the many existing results related to zero and typical cells from random tessellations. Instead, we focus here on the basic first calculations which do not require much knowledge on Poisson point processes. For more information and details, we refer the reader to the reference books [92], [114, Section 10.4] and to the survey [23]. 


\subsubsection{The zero-cell of a Poisson hyperplane tessellation}

We start with the calculation of the probability for a certain convex body $K$ to be included in $C_{o}$ the zero-cell or the typical cell. In the case of the planar Poisson line tessellation, let $K$ be a convex body containing the origin - if not, $K$ can be replaced by the convex hull of $K \cup\{o\}$. Since the number of lines from the Poisson line process intersecting $K$ is a Poisson variable with mean $\lambda \mu_{2}(K)$, we get

$$
\begin{aligned}
P\left(K \subset C_{o}\right) & =P\left(\left\{L_{\rho, \theta} \cap K=\emptyset \forall L_{\rho, \theta} \in \mathscr{P}_{\lambda}\right)\right. \\
& =\exp \left(-\mu_{2}(K)\right) \\
& =\exp (-\mathscr{L}(\partial K))
\end{aligned}
$$

where the last equality comes from the Cauchy-Crofton formula (3) and is obviously reminiscent of Buffon's needle problem. For $d \geq 3$, we get similarly, thanks to (7) applied to $k=1$ and $l=0$,

$$
P\left(K \subset C_{o}\right)=\exp \left(-\mu_{d}(K)\right)=\exp \left(-\kappa_{d-1} V_{1}(K)\right) .
$$

The use of Crofton formula for deriving the probability $P\left(K \subset C_{o}\right)$ may explain why the zero-cell of the isotropic and stationary Poisson hyperplane tessellation is often referred to as the Crofton cell.

Applying (14) to $K=B_{r}(o), r>0$, and using the equality $V_{1}\left(\mathbb{B}^{d}\right)=\frac{d \kappa_{d}}{\kappa_{d-1}}$, we obtain that the radius of the largest ball centered at $o$ and included in $C_{o}$ is exponentially distributed with mean $\left(d \kappa_{d}\right)^{-1}$.

\subsubsection{The typical cell of a Poisson hyperplane tessellation}

Let us denote by $f_{0}(\cdot)$ the number of vertices of a convex polytope. In the planar case, some general considerations show without much calculation that $E\left(f_{0}(\mathscr{C})\right)$ is equal to 4 . Indeed, we have already noted that with probability one, any vertex of a cell belongs to exactly 4 cells and is the highest point of a unique cell. Consequently, there are as many cells as vertices. In particular, the mean $\frac{1}{N_{r}} \sum_{C \in \mathbf{C}_{r}} f_{0}(C)$ is equal, up to boundary effects going to zero when $r \rightarrow \infty$, to 4 times the ratio of the total number of vertices in $B_{r}(o)$ over the total number of cells included in $B_{r}(o)$, i.e. converges to 4 . Other calculations of means and further moments of geometric characteristics can be found notably in [80, Chapter 6] and in [85, 86, 87].

One of the very few explicit distributions is the law of the inradius of $\mathscr{C}$, i.e. the radius of the largest ball included in $\mathscr{C}$. Remarkably, this radius is equal in distribution to the radius of the largest ball centered at $o$ and included in $C_{o}$, i.e. is exponentially distributed with mean $\left(d \kappa_{d}\right)^{-1}$. This result is due to R. E. Miles in dimension two and is part of an explicit construction of the typical cell $\mathscr{C}$ based on its inball [88], which has been extended to higher dimension since then, see e.g. [24]. 


\subsubsection{The typical cell of a Poisson-Voronoi tessellation}

In this subsection, we use the realization of the typical cell $\mathscr{C}$ of a Poisson-Voronoi tessellation as the cell $C\left(o \mid \mathscr{P}_{\lambda} \cup\{o\}\right)$, as explained at the end of Subsection 2.3. We follow the same strategy as for the zero-cell of a Poisson hyperplane tessellation, i.e. calculating the probability for a convex body $K$ to be contained in $\mathscr{C}$ and deducing from it the distribution of the inradius of $\mathscr{C}$.

Let $K$ be a convex body containing the origin. The set $K$ is contained in $C\left(o \mid \mathscr{P}_{\lambda} \cup\right.$ $\{o\})$ if and only if $o$ is the nearest nucleus to any point in $K$, which means that for every $x \in K$, the ball $B_{\|x\|}(x)$ does not intersect $\mathscr{P}_{\lambda}$. Let us consider the set

$$
\mathscr{F}_{o}(K)=\bigcup_{x \in K} B_{\|x\|}(x)
$$

that we call the Voronoi flower of $K$ with respect to $o$, see Figure 5.

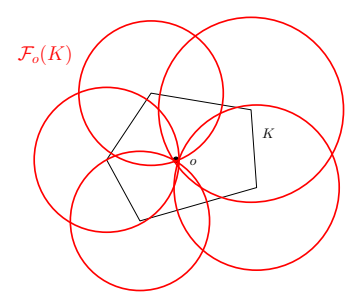

Fig. 5 Voronoi Flower (red) of the convex body $K$ (black) with respect to $o$.

Using the fact that the number of points of $\mathscr{P}_{\lambda}$ in $\mathscr{F}_{o}(K)$ is Poisson distributed with mean $\lambda V_{d}\left(\mathscr{F}_{o}(K)\right)$, we get

$$
P\left(K \subset C\left(o \mid \mathscr{P}_{\lambda} \cup\{o\}\right)\right)=\exp \left(-\lambda V_{d}\left(\mathscr{F}_{o}(K)\right)\right)
$$

Applying this to $K=B_{o}(r), r>0$, we deduce that the radius of the largest ball centered at $o$ and included in $C\left(o \mid \mathscr{P}_{\lambda} \cup\{o\}\right)$ is Weibull distributed with tail probability equal to $\exp \left(-\lambda 2^{d} \kappa_{d} r^{d}\right), r>0$.

Similarly to the case of the typical cell of a Poisson line tessellation, some direct arguments lead us to the calculation of $E\left(f_{0}(\mathscr{C})\right)$ in dimension two: any vertex of a cell belongs to exactly 3 cells and with probability one, is the either highest or lowest point of a unique cell. Consequently, there are as twice as many vertices as cells. In particular, the mean $\frac{1}{N_{r}} \sum_{C \in \mathbf{C}_{r}} f_{0}(C)$ is equal, up to boundary effects going to zero when $r \rightarrow \infty$, to 3 times the ratio of the total number of vertices in $B_{r}(o)$ over the total number of cells included in $B_{r}(o)$, i.e. equal to 6 . This means that $E\left(f_{0}(\mathscr{C})\right)=6$.

In the next section, we describe a different way of generating random polytopes: they are indeed constructed as convex hulls of random sets of points. 


\section{From Sylvester's four-point problem to random polytopes}

This section is centered around Sylvester's four-point problem, another historical problem of geometric probability which seemingly falls under the denomination of recreational mathematics but in fact lays the foundations of an active domain of today's stochastic geometry, namely the theory of random polytopes. We aim at describing first Sylvester's original question and some partial answers to it. We then survey the topic of random polytopes which has been largely investigated since the sixties, partly due to the simultaneous development of computational geometry.

\subsection{Starting from Sylvester's four-point problem}

In 1864, J. J. Sylvester published in The Educational Times [125] a problem which is nowadays known under the name of Sylvester's four-point problem and can be rephrased in the following way: given a convex body $K$ in the plane, what is the probability that 4 random points inside $K$ are the vertices of a convex quadrilateral?
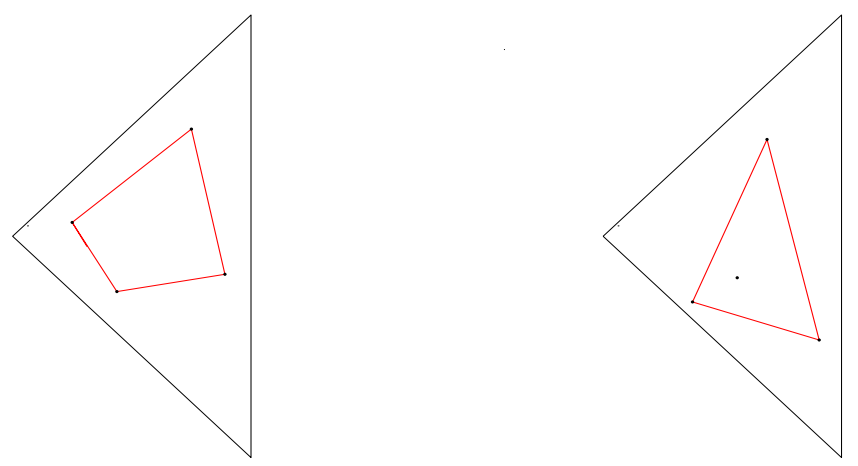

Fig. 6 Simulation of Sylvester's four-point problem in the triangle: the convex hull of the 4 uniform points (red) is either a convex quadrilateral (left) or a triangle (right).

Let us denote by $p_{4}(K)$ the probability that 4 random points which are independent and uniformly distributed in $K$ are the vertices of a convex quadrilateral. If not, one of the 4 points is included in the triangle whoses vertices are the 3 other points. Denoting by $\bar{A}(K)$ the mean area of a random triangle whoses vertices are 3 i.i.d. uniform points in $K$, we get the identity

$$
p_{4}(K)=1-4 \frac{\bar{A}(K)}{V_{2}(K)} \text {. }
$$


Solving Sylvester's problem is then equivalent to calculating the mean value $\bar{A}(K)$. In particular, the quantity $\frac{\bar{A}(K)}{V_{2}(K)}$ is scaling invariant and also invariant under any area-preserving affine transformation.

\subsubsection{Calculation of Sylvester's probability in the case of the disk}

In this subsection, we provide an explicit calculation of $p_{4}(\mathbb{D})$ where $\mathbb{D}$ is the unit disk. As in the next subsection, we follow closely the method contained in [70, pages 42-46].

Step 1. We can assume that one of the points is on the boundary of the disk. Indeed, isolating the farthest point from the origin, we get

$$
\left.V_{2}(\mathbb{D})^{3} \bar{A}(\mathbb{D})=3 \int_{\mathbb{D}}\left[\iint_{\mathbb{D}^{2}} \mathbf{1}_{\left\{\left\|x_{1}\right\|,\left\|x_{2}\right\| \in\left(0,\left\|x_{3}\right\|\right)\right\}} V_{2}\left(\operatorname{Conv}\left(\left\{x_{1}, x_{2}, x_{3}\right\}\right)\right)\right) \mathrm{d} z_{1} \mathrm{~d} z_{2}\right] \mathrm{d} z_{3} .
$$

Now for a fixed $z_{3} \in \mathbb{D} \backslash\{o\}$, we apply the change of variables $z_{i}^{\prime}=\frac{z_{i}}{\left\|z_{3}\right\|}, i=1,2$, in the double integral and we deduce that

$$
\left.\bar{A}(\mathbb{D})=\frac{3}{\pi^{3}} \int_{\mathbb{D}}\left\|z_{3}\right\|^{6}\left[\iint_{\mathbb{D}^{2}} V_{2}\left(\operatorname{Conv}\left(\left\{z_{1}^{\prime}, z_{2}^{\prime}, \frac{z_{3}}{\left\|z_{3}\right\|}\right\}\right)\right)\right) \mathrm{d} z_{1}^{\prime} \mathrm{d} z_{2}^{\prime}\right] \mathrm{d} z_{3} .
$$

Since the double integral above does not depend on $z_{3}$, we get

$$
\bar{A}(\mathbb{D})=\frac{3}{4 \pi^{2}} I
$$

where

$$
I=\iint_{\mathbb{D}^{2}} V_{2}\left(\operatorname{Conv}\left(\left\{z_{0}, z_{1}, z_{2}\right\}\right)\right) \mathrm{d} z_{1} \mathrm{~d} z_{2}
$$

$z_{0}$ being a fixed point on the boundary of $\mathbb{D}$.

Step 2. Let us now calculate $I$, i.e. $\pi^{2}$ times the mean area of a random triangle with one deterministic vertex on the unit circle and two random vertices independent and uniformly distributed in $\mathbb{D}$.

For sake of simplicity, we replace the unit disk $\mathbb{D}$ by its translate $\mathbb{D}+(0,1)$ and the fixed point on the boundary of $\mathbb{D}+(0,1)$ is chosen to be equal to $o$. This does not modify the integral $I$. The polar coordinates $\left(\rho_{i}, \theta_{i}\right)$ of $z_{i}, i=1,2$, satisfy $\rho_{i} \in$ $\left(0,2 \sin \left(\theta_{i}\right)\right)$ and

$$
V_{2}\left(\operatorname{Conv}\left(\left\{o, z_{1}, z_{2}\right\}\right)\right)=\frac{1}{2} \rho_{1} \rho_{2}\left|\sin \left(\theta_{2}-\theta_{1}\right)\right| .
$$

Consequently, we obtain 


$$
\begin{aligned}
I & =\iint_{0<\theta_{1}<\theta_{2}<\pi}\left[\int_{0}^{\left.2 \sin \left(\theta_{1}\right)\right)} \rho_{1}^{2} \mathrm{~d} \rho_{1} \int_{0}^{2 \sin \left(\theta_{2}\right)} \rho_{2}^{2} \mathrm{~d} \rho_{2}\right] \sin \left(\theta_{2}-\theta_{1}\right) \mathrm{d} \theta_{1} \mathrm{~d} \theta_{2} \\
& =\frac{64}{9} \iint_{0<\theta_{1}<\theta_{2}<\pi} \sin ^{3}\left(\theta_{1}\right) \sin ^{3}\left(\theta_{2}\right) \sin \left(\theta_{2}-\theta_{1}\right) \mathrm{d} \theta_{1} \mathrm{~d} \theta_{2} \\
& =\frac{35 \pi}{36} .
\end{aligned}
$$

Conclusion. Combining this result with (17) and (15), we get

$$
p_{4}(\mathbb{D})=1-\frac{35}{12 \pi^{2}} \approx 0.70448 \ldots
$$

\subsubsection{Calculation of Sylvester's probability in the case of the triangle}

In this subsection, we calculate the probability $p_{4}(\mathbb{T})$ where the triangle $\mathbb{T}$ is the convex hull of the three points $o,(1,1)$ and $(1,-1)$. We recall that the calculation of $p_{4}(K)$ is invariant under any area-preserving affine transformation.

Step 1. We can assume that one of the points is on the edge facing $o$. Indeed, denoting by $\left(x_{i}, y_{i}\right)$ the Cartesian coordinates of $z_{i}, i=1,2,3$, we get

$$
V_{2}(\mathbb{T})^{3} \bar{A}(\mathbb{T})=3 \int_{\mathbb{T}}\left[\iint_{\mathbb{T}^{2}} \mathbf{1}_{\left\{x_{1}, x_{2} \in\left(0, x_{3}\right)\right\}} V_{2}\left(\operatorname{Conv}\left(\left\{z_{1}, z_{2}, z_{3}\right\}\right)\right) \mathrm{d} z_{1} \mathrm{~d} z_{2}\right] \mathrm{d} z_{3} .
$$

Now for a fixed $z_{3} \in \mathbb{T} \backslash\{o\}$, we apply the change of variables $z_{i}^{\prime}=\frac{z_{i}}{x_{3}}, i=1,2$, in the double integral. We get

$$
\begin{aligned}
\bar{A}(\mathbb{T}) & =3 \int_{x_{3}=0}^{1} \int_{y_{3}=-x_{3}}^{x_{3}}\left[\iint_{\mathbb{T}^{2}} x_{3}^{6} V_{2}\left(\operatorname{Conv}\left(\left\{z_{1}^{\prime}, z_{2}^{\prime}, \frac{z_{3}}{x_{3}}\right\}\right)\right) \mathrm{d} z_{1}^{\prime} \mathrm{d} z_{2}^{\prime}\right] \mathrm{d} z_{3} \\
& =6 \int_{x_{3}=0}^{1} \int_{y_{3}=0}^{x_{3}} x_{3}^{6}\left[\iint_{\mathbb{T}^{2}} V_{2}\left(\operatorname{Conv}\left(\left\{z_{1}^{\prime}, z_{2}^{\prime}, \frac{x_{3}}{x_{3}}\right\}\right)\right) \mathrm{d} z_{1}^{\prime} \mathrm{d} z_{2}^{\prime}\right] \mathrm{d} z_{3} .
\end{aligned}
$$

Finally, for fixed $x_{3}$, we apply the change of variables $h=\frac{y_{3}}{x_{3}}$. Since the double integral in square brackets above does not depend on $x_{3}$, we get

$$
\begin{aligned}
\bar{A}(\mathbb{T}) & =6 \int_{0}^{1} x_{3}^{7} \mathrm{~d} x_{3} \int_{h=0}^{1}\left[\iint_{\mathbb{T}^{2}} V_{2}\left(\operatorname{Conv}\left(\left\{z_{1}, z_{2},(1, h)\right\}\right)\right) \mathrm{d} z_{1} \mathrm{~d} z_{2}\right] \mathrm{d} h \\
& =\frac{3}{4} \int_{h=0}^{1} I^{\prime}(h) \mathrm{d} h
\end{aligned}
$$

where $I^{\prime}(h)=\iint_{\mathbb{T}^{2}} V_{2}\left(\operatorname{Conv}\left(\left\{z_{1}, z_{2},(1, h)\right\}\right)\right) \mathrm{d} z_{1} \mathrm{~d} z_{2}$.

Step 2. Let us now calculate $I^{\prime}(h), 0<h<1$, i.e. the mean area of a random triangle with one deterministic vertex at $(1, h)$ on the vertical edge and two random vertices independent and uniformly distributed in $\mathbb{T}$. The point $(1, h)$ divides $\mathbb{T}$ into two sub- 
triangles, the upper triangle $T_{+}(h)=\operatorname{Conv}(\{o,(1, h),(1,1)\})$ and the lower triangle $T_{-}(h)=\operatorname{Conv}(\{o,(1, h),(1,-1)\})$. Let us rewrite $I^{\prime}(h)$ as

$$
I^{\prime}(h)=2 I_{+,-}^{\prime}(h)+V_{2}\left(T_{+}(h)\right)^{2} \widehat{A}\left(T_{+}(h)\right)+V_{2}\left(T_{-}(h)\right)^{2} \widehat{A}\left(T_{-}(h)\right)
$$

where

$$
\left.I_{+,-}^{\prime}(h)=\int_{z_{1} \in T_{+}(h), z_{2} \in T_{-}(h)} V_{2}\left(\operatorname{Conv}\left(\left\{z_{1}, z_{2},(1, h)\right\}\right)\right)\right) \mathrm{d} z_{1} \mathrm{~d} z_{2}
$$

and $\widehat{A}(T)$, for a triangle $T$, is the mean area of a random triangle which shares a common vertex with $T$ and has two independent vertices uniformly distributed in $T$.

We start by making explicit the quantity $\widehat{A}(T)$ for any triangle $T$. It is invariant under any area-preserving affine transformation and is multiplied by $\lambda^{2}$ when $T$ is rescaled by $\lambda^{-1}$. Consequently, it is proportional to $V_{2}(T)$, i.e.

$$
\widehat{A}(T)=\widehat{A}(\mathbb{T}) V_{2}(T) .
$$

We now calculate

$$
\widehat{A}(\mathbb{T})=\int_{\mathbb{T}^{2}} V_{2}\left(\operatorname{Conv}\left(\left\{o, z_{1}, z_{2}\right\}\right)\right) \mathrm{d} z_{1} \mathrm{~d} z_{2}
$$

The polar coordinates $\left(\rho_{i}, \theta_{i}\right)$ of $x_{i}, i=1,2$, satisfy $\rho_{i} \in\left(0, \cos ^{-1}\left(\theta_{i}\right)\right)$ and equality (18). Consequently, we obtain

$$
\begin{aligned}
\widehat{A}(\mathbb{T}) & =\int_{-\frac{\pi}{4}}^{\frac{\pi}{4}} \int_{-\frac{\pi}{4}}^{\frac{\pi}{4}}\left[\int_{0}^{\cos ^{-1}\left(\theta_{1}\right)} \rho_{1}^{2} \mathrm{~d} \rho_{1} \int_{0}^{\cos ^{-1}\left(\theta_{2}\right)} \rho_{2}^{2} \mathrm{~d} \rho_{2}\right] \sin \left(\theta_{2}-\theta_{1}\right) \mathrm{d} \theta_{1} \mathrm{~d} \theta_{2} \\
& =\frac{1}{9} \iint_{-\frac{\pi}{4}<\theta_{1}<\theta_{2}<\frac{\pi}{4}} \frac{\sin \left(\theta_{2}-\theta_{1}\right)}{\cos ^{3}\left(\theta_{1}\right) \cos ^{3}\left(\theta_{2}\right)} \mathrm{d} \theta_{1} \mathrm{~d} \theta_{2} \\
& =\frac{4}{27} .
\end{aligned}
$$

Combining (22) and (23), we obtain in particular that

$$
\widehat{A}\left(T_{+}(h)\right)=\frac{2(1-h)}{27} \text {, and } \widehat{A}\left(T_{-}(h)\right)=\frac{2(1+h)}{27} .
$$

We turn now our attention to the quantity $I_{+,-}^{\prime}(h)$ defined at (21). Using the rewriting of the area of a triangle as half of the non-negative determinant of two vectors and introducing $g_{+}(h)$ (resp. $g_{-}(h)$ ) as the center of mass of $T_{+}(h)$ (resp. $T_{-}(h)$ ), we obtain 


$$
\begin{aligned}
I_{+,-}^{\prime}(h) & =\frac{1}{2} \int_{z_{1} \in T_{+}(h), z_{2} \in T_{-}(h)} \operatorname{det}\left(z_{1}-(1, h), z_{2}-(1, h)\right) \mathrm{d} z_{1} \mathrm{~d} z_{2} \\
& =\frac{1}{2} \int_{z_{1} \in T_{+}(h)} \operatorname{det}\left(\left(z_{1}-(1, h)\right), \int_{z_{2} \in T_{-}(h)}\left(z_{2}-(1, h)\right)\right) \mathrm{d} z_{1} \\
& =\frac{V_{2}\left(T_{-}(h)\right)}{2} \operatorname{det}\left(\int_{z_{1} \in T_{+}(h)}\left(z_{1}-(1, h)\right) \mathrm{d} z_{1},\left(g_{-}(h)-(1, h)\right)\right) \\
& =V_{2}\left(T_{-}(h)\right) V_{2}\left(T_{+}(h)\right) V_{2}\left(\operatorname{Conv}\left(\left\{g_{+}(h), g_{-}(h),(1, h)\right\}\right)\right) \\
& =\frac{(1-h)(1+h)}{4} \frac{V_{2}(\mathbb{T})}{9}=\frac{1-h^{2}}{36} .
\end{aligned}
$$

Inserting (24) and (25) into (20), we get

$$
I^{\prime}(h)=\frac{1-h^{2}}{18}+\frac{(1-h)^{3}}{54}+\frac{(1+h)^{3}}{54}=\frac{5+3 h^{2}}{54} .
$$

Conclusion. Combining (26) with (19) and (15), we get $\bar{A}(\mathbb{T})=\frac{1}{12}$ and

$$
p_{4}(T)=\frac{2}{3}=0.66666 \ldots
$$

\subsubsection{Extremes of $p_{4}(K)$}

In 1917, W. Blaschke proved a monotonicity result for Sylvester's four-point problem, namely that the probability $p_{4}(K)$ is maximal when $K$ is a disk and minimal when $K$ is a triangle, see [15] and [16, $24, \S 25]$. Because of (15), this amounts to saying that the mean area of a random triangle in a unit area convex body $K$ is minimal when $K$ is a disk and maximal when $K$ is a triangle.

This assertion is due to the use of symmetrization techniques which have become classical since then in convex and integral geometry. We describe below the main arguments developed by $\mathrm{W}$. Blaschke and also rephrased in a nice way in the historical note [100].

Let $K$ be a unit area convex body of $\mathbb{R}^{2}$ and let us denote by $x_{\min }$ and $x_{\max }$ the minimal and maximal projection on the $x$-axis of a point of $K$. The boundary of $K$ is parametrized by two functions $f_{+}, f_{-}:\left[x_{\min }, x_{\max }\right] \longrightarrow \mathbb{R}$ such that $f_{+} \geq f_{-}$with equality at $x_{\min }$ and $x_{\max }$. We use the term Steiner symmetrization of $K$ with respect to the $x$-axis for the transformation

$$
\mathscr{S}:\left\{\begin{array}{l}
K \longrightarrow \mathbb{R}^{2} \\
(x, y) \longmapsto\left(x, y-\frac{f_{+}(x)+f_{-}(x)}{2}\right)
\end{array}\right.
$$

In other words, $\mathscr{S}$ sends any segment which is the intersection of $K$ with a vertical line to its unique vertical translate which is symmetric with respect to the $x$-axis; see Figure 7. In particular, $\mathscr{S}$ is area-preserving and the image of $K$ is a convex body which is symmetric with respect to the $x$-axis, see e.g. [110, Section 10.3]. Let us 
show that

$$
\bar{A}(K) \geq \bar{A}(\mathscr{S}(K))
$$

Let $z_{i}=\left(x_{i}, y_{i}\right), i=1,2,3$, be three points of $K$. In particular, we start by noticing that the area of the parallelogram spanned by the two vectors $\left(z_{2}-z_{1}\right)$ and $\left(z_{3}-z_{1}\right)$ is twice the area of the triangle $\operatorname{Conv}\left(\left\{z_{1}, z_{2}, z_{3}\right\}\right)$. Consequently, we get

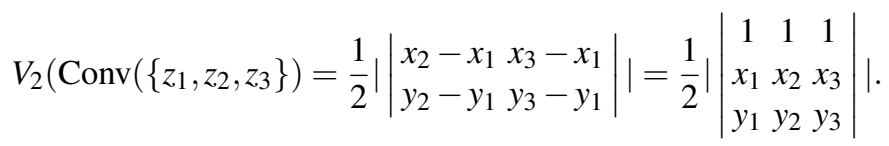

We will also use the points $z_{i}^{*}=\mathscr{S}\left(z_{i}\right)=\left(x_{i}, y_{i}^{*}\right), \overline{z_{i} *}=\left(x_{i},-y_{i}^{*}\right)$ and $w_{i}=\left(x_{i}, y_{i}-\right.$ $\left.2 y_{i}^{*}\right), i=1,2,3$. In particular, the identity $\mathscr{S}\left(w_{i}\right)=\overline{z_{i} *}$ is satisfied and the two triangles $\operatorname{Conv}\left(\left\{z_{1}, z_{2}, z_{3}\right\}\right)$ and $\operatorname{Conv}\left(\left\{w_{1}, w_{2}, w_{3}\right\}\right)$ have same area. Consequently,

$$
\begin{aligned}
V_{2}\left(\operatorname{Conv}\left(\left\{z_{1}, z_{2}, z_{3}\right\}\right)+V_{2}\left(\operatorname{Conv}\left(\left\{w_{1}, w_{2}, w_{3}\right\}\right)\right.\right. \\
\quad \geq \frac{1}{2}|| \begin{array}{ccc}
1 & 1 & 1 \\
x_{1} & x_{2} & x_{3} \\
y_{1} & y_{2} & y_{3}
\end{array}|-| \begin{array}{ccc}
1 & 1 & 1 \\
x_{1} & x_{2} & x_{3} \\
y_{1}-2 y_{1}^{*} & y_{2}-2 y_{2}^{*} & y_{3}-2 y_{3}^{*}
\end{array}|| \\
\geq \frac{1}{2}\left|\begin{array}{ccc}
1 & 1 & 1 \\
x_{1} & x_{2} & x_{3} \\
2 y_{1}^{*} & 2 y_{2}^{*} & 2 y_{3}^{*}
\end{array}\right| \mid \\
=2 V_{2}\left(\operatorname{Conv}\left(\left\{z_{1}^{*}, z_{2}^{*}, z_{3}^{*}\right\}\right)\right) .
\end{aligned}
$$

Integrating (29) with respect to $z_{1}, z_{2}, z_{3} \in K$ and using the fact that both the transformation $\mathscr{S}$ and the reflection with respect to the $x$-axis preserve the Lebesgue measure, we obtain (27). It remains to use the fact that the equality in (27) is satisfied only when $K$ is an ellipse. In fact, for any convex body $K$, there exists a sequence of lines such that the image of $K$ under consecutive applications of Steiner symmetrizations with respect to the lines of that sequence converges to a disk [26]. The function $\bar{A}(\cdot)$ being continuous on the set of convex bodies, we obtain $\bar{A}(K) \geq \bar{A}\left(\frac{1}{\sqrt{\pi}} \mathbb{D}\right)$ and therefore $p_{4}(K) \leq p_{4}(\mathbb{D})$.

We turn now our attention to the proof of $p_{4}(K) \leq p_{4}(\mathbb{T})$. The method relies on a transformation $\mathscr{T}$ in the same spirit as the Steiner symmetrization, called Schüttelung or shaking and defined as follows:

$$
\mathscr{T}::\left\{\begin{array}{l}
K \longrightarrow \mathbb{R}^{2} \\
(x, y) \longmapsto\left(x, y-f_{-}(x)\right)
\end{array}\right.
$$

In other words, $\mathscr{T}$ sends any segment which is the intersection of $K$ with a vertical line to its unique vertical translate with a lower-end on the $x$-axis, see Figure 7 . In particular, $\mathscr{T}$ is area-preserving and preserves the convexity.

Using both (28) and the fact that $K$ is a unit-area convex body which satisfies the equality

$$
K=\left\{(x, y): x \in\left[x_{\min }, x_{\max }\right], f_{-}(x) \leq y \leq f_{+}(x)\right\}
$$



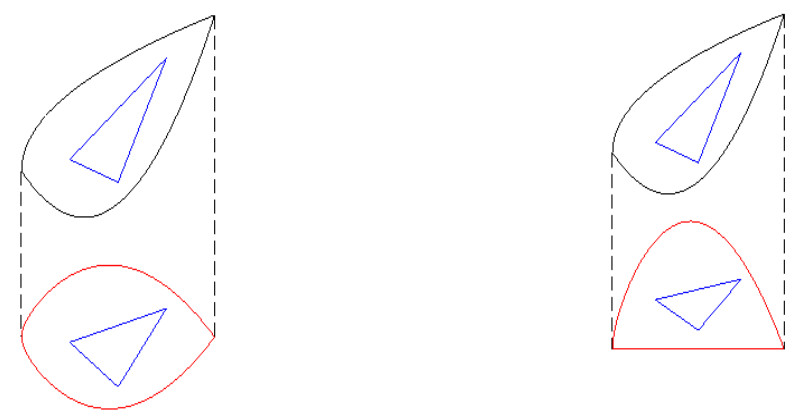

Fig. 7 Two symmetrizations (red) of a convex body delimited by translates of the curves $f_{+}(x)=$ $\sqrt{x}$ and $f_{-}(x)=x\left(x-2+\frac{\sqrt{2}}{2}\right)$ on the interval $[0,2]$ (black) and the image of a triangle by symmetrization (blue): the Steiner symmetrization (left) and the shaking (right).

we get that

$$
\bar{A}(K)=\iiint_{\left[x_{\min }, x_{\max }\right]} I\left(x_{1}, x_{2}, x_{3}\right) \mathrm{d} x_{1} \mathrm{~d} x_{2} \mathrm{~d} x_{3}
$$

where

$$
\begin{aligned}
& I\left(x_{1}, x_{2}, x_{3}\right) \\
& =\int_{f_{-}\left(x_{1}\right)}^{f_{+}\left(x_{1}\right)} \int_{f_{-}\left(x_{2}\right)}^{f_{+}\left(x_{2}\right)} \int_{f_{-}\left(x_{3}\right)}^{f_{+}\left(x_{3}\right)} \frac{1}{2}|| \begin{array}{ccc}
1 & 1 & 1 \\
x_{1} & x_{2} & x_{3} \\
y_{1} & y_{2} & y_{3}
\end{array}|| \mathrm{d} y_{3} \mathrm{~d} y_{2} \mathrm{~d} y_{1} \\
& =\int_{f_{-}\left(x_{1}\right)}^{f_{+}\left(x_{1}\right)} \int_{f_{-}\left(x_{2}\right)}^{f_{+}\left(x_{2}\right)} \int_{f_{-}\left(x_{3}\right)}^{f_{+}\left(x_{3}\right)} \frac{1}{2}\left|a_{1} y_{1}+a_{2} y_{2}+a_{3} y_{3}\right| \mathrm{d} y_{3} \mathrm{~d} y_{2} \mathrm{~d} y_{1} .
\end{aligned}
$$

and with $a_{1}=a_{1}\left(x_{1}, x_{2}, x_{3}\right)=\left(x_{3}-x_{2}\right), a_{2}=a_{2}\left(x_{1}, x_{2}, x_{3}\right)=\left(x_{1}-x_{3}\right)$ and $a_{3}=$ $a_{3}\left(x_{1}, x_{2}, x_{3}\right)=\left(x_{2}-x_{1}\right)$. For sake of simplicity, the dependency of the coefficients $a_{i}$ on the coordinates $x_{i}$ is omitted. When $x_{1}, x_{2}, x_{3}$ are fixed, the function $I$ calculates the 4-dimensional volume of the parallelepiped region delimited by the rectangular basis $\left[f_{-}\left(x_{1}\right), f_{+}\left(x_{1}\right)\right] \times\left[f_{-}\left(x_{2}\right), f_{+}\left(x_{2}\right)\right] \times\left[f_{-}\left(x_{3}\right), f_{+}\left(x_{3}\right)\right] \times\{0\}$ and the surface of equation $y_{4}=\frac{1}{2}\left|a_{1} y_{1}+a_{2} y_{2}+a_{3} y_{3}\right|$. In particular, if we allow the rectangular basis to be translated, the integral $I$ only depends on the distance $\mathscr{D}$ in $\mathbb{R}^{3}$ from the midpoint $\left(\frac{f_{-}\left(x_{1}\right)+f_{+}\left(x_{1}\right)}{2}, \frac{f_{-}\left(x_{2}\right)+f_{+}\left(x_{2}\right)}{2}, \frac{f_{-}\left(x_{3}\right)+f_{+}\left(x_{3}\right)}{2}\right)$ of the rectangular basis to the set $\left\{\left(y_{1}, y_{2}, y_{3}\right): a_{1} y_{1}+a_{2} y_{2}+a_{3} y_{3}=0\right\}$ and is even an increasing function of $\mathscr{D}$. We notice that $\mathscr{D}$ satisfies 


$$
\begin{aligned}
\mathscr{D} & =\frac{\left|a_{1}\left(f_{-}\left(x_{1}\right)+f_{+}\left(x_{1}\right)\right)+a_{2}\left(f_{-}\left(x_{2}\right)+f_{+}\left(x_{2}\right)\right)+a_{3}\left(f_{-}\left(x_{3}\right)+f_{+}\left(x_{3}\right)\right)\right|}{2 \sqrt{a_{1}^{2}+a_{2}^{2}+a_{3}^{2}}} \\
& =\frac{1}{2 \sqrt{a_{1}^{2}+a_{2}^{2}+a_{3}^{2}}}\left|\begin{array}{ccc}
1 & 1 & 1 \\
x_{1} & x_{2} & x_{3} \\
f_{-}\left(x_{1}\right) & f_{-}\left(x_{2}\right) & f_{-}\left(x_{3}\right)
\end{array}\right|+\left|\begin{array}{ccc}
1 & 1 & 1 \\
x_{1} & x_{2} & x_{3} \\
f_{+}\left(x_{1}\right) & f_{+}\left(x_{2}\right) & f_{+}\left(x_{3}\right)
\end{array}\right| \mid .
\end{aligned}
$$

Now, the two determinants in the last equality above are two times the algebraic areas of two triangles whose vertices are on $\partial K$ and have respective $x$-coordinates $x_{1}, x_{2}$ and $x_{3}$. Because of the convexity of $K$, they must have opposite signs. Consequently, we get from the triangular inequality ||$a|-| b|| \leq|a-b|$ that

$$
\mathscr{D} \leq \frac{1}{2 \sqrt{a_{1}^{2}+a_{2}^{2}+a_{3}^{2}}}|| \begin{array}{ccc}
1 & 1 & 1 \\
x_{1} & x_{2} & x_{3} \\
f_{+}\left(x_{1}\right)-f_{-}\left(x_{1}\right) & f_{+}\left(x_{2}\right)-f_{-}\left(x_{2}\right) & f_{+}\left(x_{3}\right)-f_{-}\left(x_{3}\right)
\end{array}|| .
$$

In particular, after application of the transformation $\mathscr{T}$, the distance is equal to the right-hand side of the inequality above. The integral $I\left(x_{1}, x_{2}, x_{3}\right)$ being an increasing function of $\mathscr{D}$, it is greater, which implies thanks to (30) that

$$
\bar{A}(K) \leq \bar{A}(\mathscr{T}(K)) .
$$

It remains to use the fact that there exists a sequence of lines such that the image of $K$ under consecutive applications of the Schüttelung operations with respect to these lines converges to a triangle [13]. Therefore, we get the inequality $\bar{A}(K) \leq \bar{A}(\mathbb{T})$ and thanks to (15), the required inequality $p_{4}(K) \geq p_{4}(\mathbb{T})$.

\subsection{Random polytopes}

There are several ways of extending Sylvester's initial question:

(a)increasing the number of random points inside $K$ and ask for the probability that $n$ i.i.d. points uniformly distributed in a two-dimensional convex body $K$ are in convex position, i.e. are extreme points of their convex hull;

(b)increasing the dimension and ask for the probability that $(d+2)$ or more i.i.d. points uniformly distributed in a $d$-dimensional convex body $K$ are the vertices of a convex polytope;

(c) increasing the number of random points in any dimension and ask more general questions, such as the distribution and mean value of the number of extreme points and of other characteristics of the convex hull;

(d)replacing the uniform distribution in $K$ by another probability distribution in $\mathbb{R}^{d}$.

The topic of random polytopes has become more popular in the last 50 years and this is undoubtedly due in part to the birth of computational geometry and the need to get quantitative information on the efficiency of algorithms in discrete geometry, 
in particular algorithms designed for the construction of the convex hull of multivariate data. We describe below the state of the art on each of the problems above.

Problem (a). Let us denote by $p_{n}(K)$ the probability that $n$ i.i.d. points uniformly distributed in $K$ are in convex position. Using combinatorial arguments, P. Valtr obtained explicit calculations for $p_{n}\left([0,1]^{2}\right)[126]$ and $p_{n}(\mathbb{T})$ [127]. More recently, J.-F. Marckert provided a recursive formula for $p_{n}(\mathbb{D})$, which he implements to derive explicit values up to $p_{8}(\mathbb{D})$ [77]. Though there is no general formula for every $K$, the sequences $p_{n}(K)$ for all convex bodies $K$ share a common asymptotic behavior when $n \rightarrow \infty$. In a breakthrough paper [8] in 1999, I. Bárány showed that for every $K$ with area 1 , when $n \rightarrow \infty$,

$$
\log p_{n}(K)=-2 n \log (n)+n \log \left(\frac{1}{4} e^{2} \mathrm{pa}(K)\right)+o(n)
$$

where $f(n)=o(g(n))$ means that $\lim _{n \rightarrow \infty} f(n) / g(n)=0$ and $\mathrm{pa}(K)$ is the supremum of the so-called affine perimeter of all convex bodies included in $K$, see Section 3.2.2.

Problem (b). Let us denote by $p_{n}^{(d)}(K)$ the probability that $n$ i.i.d. points uniformly distributed in a convex body $K$ of $\mathbb{R}^{d}$ are in convex position. J. F. C. Kingman calculated $p_{d+2}^{(d)}\left(\mathbb{B}^{d}\right)$ in 1969 [71] and it was shown by H. Groemer in 1973 that $p_{d+2}^{(d)}(K)$ is minimal when $K$ is the unit ball (or an ellipsoid) [50]. It is still undecided whether the $d$-dimensional simplex should maximize $p_{d+2}^{(d)}(K)$ though $\mathrm{W}$. Blaschke had claimed that his proof in the case $d=2$ could be directly extended [15]. Regarding the asymptotic behavior of $p_{n}^{(d)}(K)$ when $n \rightarrow \infty$, I. Bárány conjectured a two-term expansion in the spirit of the two-dimensional case and showed the following one-term expansion [9]:

$$
\log p_{n}(K)=-\frac{2}{d-1} n \log (n)+O(n)
$$

where $f(n)=O(g(n))$ means that $f / g$ is bounded.

Problem (c). Let $K$ be a convex body of $\mathbb{R}^{d}$ and let $K_{n}$ be the convex hull of $n$ i.i.d. points uniformly distributed in $K$. The natural questions on this model have to do with the shape of $K_{n}$ and the distributions of the geometric characteristics of $K_{n}$ such as the number of vertices, number of faces or the volume. They can be treated in the two different contexts of fixed $n$ and $n$ large. This will be the focus of the end of the section.

Problem (d). There have been several works related to Problem (d). Wendel's result described below is one of them in the case of a symmetric distribution with respect to $o$. Other papers have focused on isotropic distributions [27] and especially the 
Gaussian distribution, see e.g. $[108,10]$.

For a more detailed account on the topic of random polytopes, we refer the reader to [114, Chapter 8], the lecture [60] and the very exhaustive survey [107]. In the rest of the section, we will present a few results related to Problem (c) in both the nonasymptotic and asymptotic regimes.

\subsubsection{Non-asymptotic results}

In this subsection, we describe two of the non-asymptotic results on the convex hull $K_{n}$ of $n$ i.i.d. points uniformly distributed in a convex body $K$ of $\mathbb{R}^{d}$ : the Efron identity relating first moments of functionals of $K_{n}$ and Wendel's calculation of the probability that the origin $o$ belongs to $K_{n}$. In the sequel, $f_{k}(\cdot)$ is the number of $k$ dimensional faces of a convex polytope. In particular, $f_{0}(\cdot)$ denotes the number of vertices.
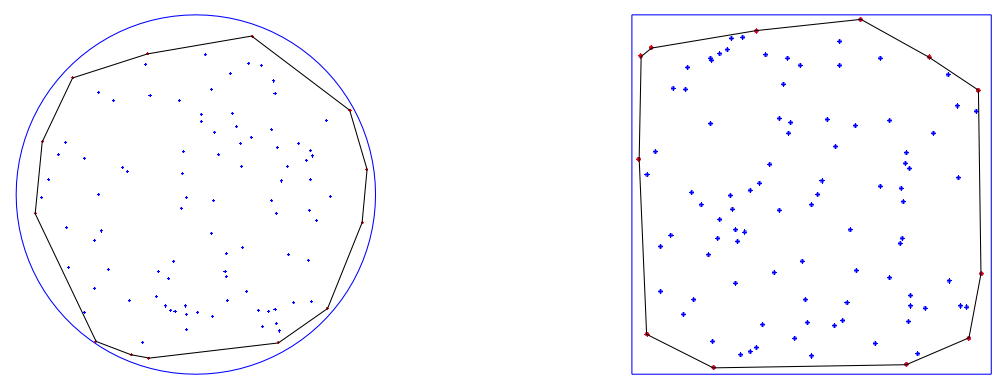

Fig. 8 Simulations of the random polytope $K_{100}$ (black) when $K$ is a disk (left) and $K$ is a square (right).

In 1965, B. Efron proved an extension of (15), i.e. he provided an identity which connects in a very simple way the mean number of vertices of the convex hull of $n$ points to the mean volume of the convex hull of $(n-1)$ points [40]. The calculation, which has been extended since then by several identities for higher moments due to C. Buchta [19], goes as follows. Let $X_{1}, \cdots, X_{n}$ be the $n$ i.i.d. uniform points in $K$. Then almost surely,

$$
f_{0}\left(K_{n}\right)=\sum_{k=1}^{n} \mathbf{1}_{\left\{X_{k} \notin \operatorname{Conv}\left(X_{1}, \cdots, X_{k-1}, X_{k+1}, \cdots, X_{n}\right)\right\}} \cdot
$$

Taking the expectation of this equality, we obtain 


$$
\begin{aligned}
E\left(f_{0}\left(K_{n}\right)\right) & =n P\left(X_{n} \notin \operatorname{Conv}\left(X_{1}, \cdots, X_{n-1}\right)\right) \\
& =n E\left(E\left(\mathbf{1}_{\left\{X_{n} \notin \operatorname{Conv}\left(X_{1}, \cdots, X_{n-1}\right)\right\}} \mid X_{1}, \cdots, X_{n-1}\right)\right. \\
& =n\left(1-\frac{E\left(V_{d}\left(K_{n-1}\right)\right)}{V_{d}(K)}\right) .
\end{aligned}
$$

In 1962, J. G. Wendel showed an explicit formula for the probability that the origin $o$ lies inside the convex hull of $n$ i.i.d. points with a symmetric distribution with respect to $o$ [131]. Let $X_{1}, \cdots, X_{n}, n \geq 1$, be the random points and we assume additionally that their common distribution is such that with probability one, all subsets of size $d$ are linearly independent. We first notice that $o$ is not in the convex hull if and only if there exists a half-space containing all the points, i.e. there exists $y \in \mathbb{R}^{d}$ such that $\left\langle y, X_{k}\right\rangle>0$ for every $1 \leq k \leq n$. This implies in particular that the probability $P\left(o \notin \operatorname{Conv}\left(\left\{X_{1}, \cdots, X_{n}\right\}\right)\right)$ equals 1 as soon as $n \leq d$ and $2^{-(n-1)}$ when $d=1$. Now the calculation for $n \geq(d+1) \geq 3$ is done by purely combinatorial arguments.

Indeed, each $X_{k}$ defines a set of authorized $y$ which is a half-space bounded by the linear hyperplane $H_{k}$ with normal vector $X_{k}$. Consequently, each connected component of the complement of $\bigcup_{k=1}^{n} H_{k}$ can be coded by a sequence in $\{-1,1\}^{n}$ where +1 at the $k$-th position means that the connected component lies in the authorized half-space bounded by $H_{k}$. There are $2^{n}$ possible codes and we denote by $N_{d, n}$ the total number of connected components. The variable $N_{d, n}$ is almost surely constant, as we shall see later on. Recalling from the earlier discussion that a necessary and sufficient condition to have the origin outside of the convex hull is that the intersection of all authorized half-spaces bounded by the hyperplanes $H_{k}$ is not empty, we obtain the following equivalence: $o$ is not in the convex hull of $\left\{X_{1}, \cdots, X_{n}\right\}$ if and only if one of the connected components is coded by $(1, \cdots, 1)$. This happens with probability

$$
P\left(o \notin \operatorname{Conv}\left(\left\{X_{1}, \cdots, X_{n}\right\}\right)=\frac{N_{d, n}}{2^{n}} .\right.
$$

As announced earlier, the variable $N_{d, n}$ is constant on the event of probability one that any subset of size $d$ of the $n$ points is linearly independent. We calculate $N_{d, n}$ on this event by proving a recurrence relation. For fixed $n \geq 2$, the $n$-th hyperplane $H_{n}$ separates into two subparts each connected component of $\mathbb{R}^{d} \backslash \bigcup_{k=1}^{n-1} H_{k}$ that it meets and leaves unchanged the remaining connected components of $\mathbb{R}^{d} \backslash \bigcup_{k=1}^{n-1} H_{k}$. The number of connected components of $\mathbb{R}^{d} \backslash \bigcup_{k=1}^{n-1} H_{k}$ that $H_{n}$ meets is equal to the number of connected components of $H_{n} \backslash \bigcup_{k=1}^{n-1} H_{k}$, i.e. $N_{d-1, n-1}$ while the number of untouched connected components of $\mathbb{R}^{d} \backslash \bigcup_{k=1}^{n-1} H_{k}$ is $N_{d, n-1}-N_{d-1, n-1}$. Consequently, we get the relation

$$
N_{d, n}=N_{d-1, n-1}+N_{d, n-1}
$$

Using that $N_{d, 1}=2$ for every $d \geq 1$, we deduce that $N_{d, n}=2 \sum_{k=0}^{d-1}\left(\begin{array}{c}n-1 \\ k\end{array}\right)$ thanks to Pascal's triangle. This last equality combined with (31) leads to 


$$
P\left(o \notin \operatorname{Conv}\left(\left\{X_{1}, \cdots, X_{n}\right\}\right)=2^{-(n-1)} \sum_{k=0}^{d-1}\left(\begin{array}{c}
n-1 \\
k
\end{array}\right) .\right.
$$

In particular, when $n \rightarrow \infty$, this probability goes to zero exponentially fast. In the next section, we investigate the general asymptotic behavior of $K_{n}$.

\subsubsection{Asymptotic results}

When $n \rightarrow \infty, K_{n}$ converges to $K$ itself and studying the asymptotic behavior of $K_{n}$ means being able to quantify the quality of the approximation of $K$ by $K_{n}$. The first breakthrough is due to A. Rényi and R. Sulanke in 1963 and 1964 [108, 109]. They showed in particular that in the planar case, the mean number of vertices of $K_{n}$ has a behavior which is highly dependent on the regularity of the boundary of $K$. Indeed, when $\partial K$ is of class $\mathscr{C}^{2}$,

$$
E\left(f_{0}\left(K_{n}\right)\right) \underset{n \rightarrow \infty}{\sim} 2^{\frac{1}{3}} 3^{-\frac{1}{3}} \Gamma\left(\frac{5}{3}\right) V_{d}(K)^{-\frac{1}{3}} \int_{\partial K} r_{s}^{-\frac{1}{3}} \mathrm{~d} s n^{\frac{1}{3}} .
$$

where $r_{s}$ is the radius of curvature of $\partial K$ at $s$ and $f(n) \underset{n \rightarrow \infty}{\sim} g(n)$ means that $f / g$ has limit 1 . The quantity $\int_{\partial K} r_{s}^{-\frac{1}{3}} \mathrm{~d} s$ is called the affine perimeter of $K$.

When $K$ is a convex polygon itself, the number of vertices of $K_{n}$ is expected to be smaller in mean as, roughly speaking, it does not require many edges to approximate the flat parts of the boundary of $K$. While the extreme points of $K_{n}$ are more or less homogeneously spread along the curve $\partial K$ when it is smooth, they are concentrated in the corners, i.e. around the vertices of $K$ when $K$ is a convex polygon. Consequently, the growth rate of $E\left(f_{0}\left(K_{n}\right)\right)$ becomes logarithmic, as opposed to the polynomial rate from (32). Denoting by $r$ the number of vertices of the convex polygon $K$, we get

$$
E\left(f_{0}\left(K_{n}\right)\right) \underset{n \rightarrow \infty}{\sim} \frac{2 r}{3} \log n .
$$

The two estimates (32) and (33) have been extended in many directions: asymptotic means of $f_{k}\left(K_{n}\right), 1 \leq k \leq d$ and of $V_{d}\left(K_{n}\right)$ in any dimension and convergences $[7,106]$, same for the intrinsic volumes in the smooth case [116, 104], concentration estimates [128], second-order results [105, 25] and so on. Many basic questions remain unanswered, like for instance the asymptotic behavior of the mean intrinsic volumes of $K_{n}$ when $K$ is a polytope.

In the next section, we generate for the first time non-convex random sets as unions of translates of a so-called grain. 


\section{From the bicycle wheel problem to random coverings and continuum percolation}

In this section, we start with a practical problem which can be reinterpreted as a random covering problem on the circle by random arcs with fixed length. We solve it and discuss possible extensions. This leads us to a classical model of random covering of the Euclidean space called the Boolean model and we present briefly some of the questions related to it: covering of a particular set, continuum percolation and shapes of the connected components of the two phases.

\subsection{Starting from the bicycle wheel problem and random covering of the circle}

In 1989, C. Domb tells how his work during the second World War in radar research for the Admiralty led him to ask H. Jeffreys in 1946 about a covering problem [38]. H. Jeffreys related his question to his own bicycle wheel problem which he had formulated several years before in the following way: a man is cycling along a road and passes through a region threwn with tacks. He wishes to know whether one has entered his tire. Because of the traffic, he can only snatch glances at random times. At each glance he has covered a fraction $x$ of the wheel. What is the probability that after $n$ glances, he has covered the whole wheel?. It turns out that the problem had been solved by W. L. Stevens in 1939 [123], see also [121, Chapter 4]. Surprisingly, his method that we describe below relies exclusively on combinatorial arguments.

We start by rephrasing the problem in mathematical terms in the following way: a set of $n$ intervals of length $x$ are placed randomly on a circle of length one and we aim at calculating the probability $q_{n}(x)$ that the circle is fully covered. The endpoints

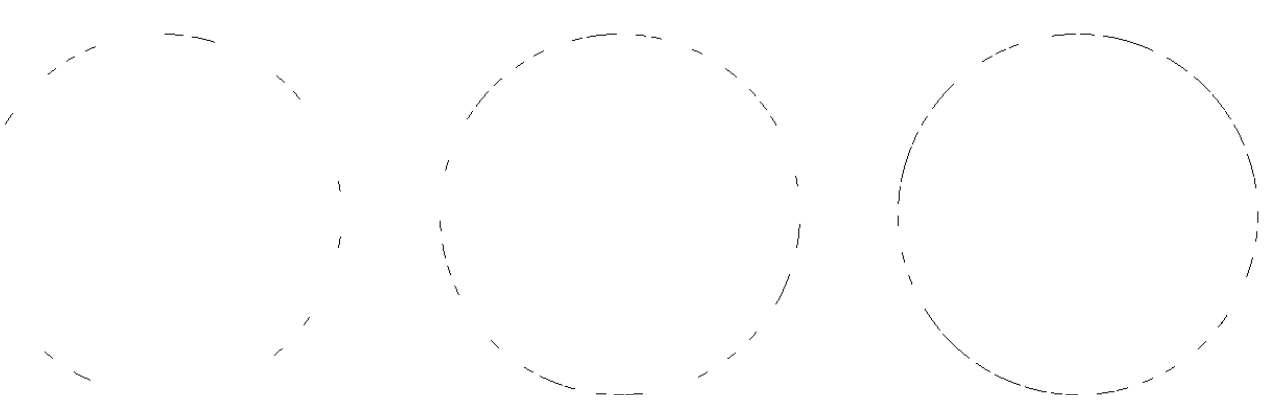

Fig. 9 Simulations of the bicycle wheel problem for $x=0.01$ : the random intervals in the cases $n=20$ (left), $n=50$ (middle) and $n=100$ (right).

in the anticlockwise direction of the $n$ random intervals of length $x$ are denoted by 
$U_{1}, \cdots, U_{n}$ and are assumed to be $n$ i.i.d. random variables uniformly distributed in $(0,1)$. For every $1 \leq i \leq n$, we consider the event denoted by $A_{i}$ that the endpoint of the $i$-th arc is not covered by the other $(n-1)$ random intervals. The key idea consists in noticing that the circle is fully covered by the $n$ random intervals if and only if all endpoints are covered. Consequently, using the inclusion-exclusion principle, we obtain that the probability $p_{\mathrm{cov}}(n, x)$ satisfies

$$
\begin{aligned}
1-p_{\text {cov }}(n, x) & =P\left(\cup_{i=1}^{n} A_{i}\right) \\
& =\sum_{k=1}^{n}(-1)^{k+1} \sum_{1 \leq i_{1}<i_{2}<\cdots<i_{k} \leq n} P\left(A_{i_{1}} \cap A_{i_{2}} \cap \cdots \cap A_{i_{k}}\right) \\
& =\sum_{k=1}^{n}(-1)^{k+1}\left(\begin{array}{l}
n \\
k
\end{array}\right) P\left(A_{1} \cap A_{2} \cap \cdots A_{k}\right),
\end{aligned}
$$

where the last equality comes from the fact that the variables $U_{1}, \cdots, U_{n}$ are exchangeable. It remains to calculate $P\left(A_{1} \cap A_{2} \cap \cdots A_{k}\right), 1 \leq k \leq n$, i.e. the probability that the endpoint of each of the first $k$ random intervals is not covered, not only by the $(k-1)$ other intervals from the first bunch but also by the $(n-k)$ remaining intervals. This means that the event $A_{1} \cap A_{2} \cap \cdots A_{k}$ can be rewritten as

$$
A_{1} \cap A_{2} \cap \cdots A_{k}=B_{k} \cap\left(\bigcap_{i=k+1}^{n} C_{i, k}\right)
$$

where $B_{k}$ is the event that the endpoint of each of the first $k$ random intervals is not covered by the $(k-1)$ other intervals from the first bunch and $C_{i, k}, k+1 \leq i \leq n$, is the event that the $i$-th random arc does not cover any of the endpoints from the first $k$ random intervals.

On the event $B_{k}$, the $k$ endpoints are at distance at least $x$ from each other. Consequently, $P\left(B_{k}\right)$ is the probability that a random division of the circle into $k$ parts produces parts of lengths larger than $x$. This is in particular the exact problem 666 that W. A. Whitworth solves in his book Choice and Chance published in 1870 [132]. By a direct integral calculation, we get

$$
P\left(B_{k}\right)=(1-k x)_{+}^{k-1} .
$$

Conditional on the positions of the first $k$ random intervals which satisfy the condition of the event $B_{k}$, the events $C_{i, k}$ are independent. The set of allowed positions on the circle for the endpoint of the $i$-th random arc is then the complement of a union of $k$ disjoint intervals of length $x$. Consequently,

$$
P\left(\bigcap_{i=k+1}^{n} C_{i, k} \mid B_{k}\right)=(1-k x)_{+}^{n-k} .
$$

Combining (36), (37) with (35) and (34) shows that 


$$
p_{\text {cov }}(n, x)(x)=\sum_{k=0}^{n}(-1)^{k}\left(\begin{array}{l}
n \\
k
\end{array}\right)(1-k x)_{+}^{n-1} .
$$

In 1982, the formula was extended by A. F. Siegel and L. Holst to the explicit calculation of $p_{\text {cov }}(n, \mu)$, i.e. the probabiity to cover the circle with i.i.d. intervals which have random lengths such that these lengths are i.i.d. $\mu$-distributed variables which are independent of the positions of the intervals on the circle [120]. They even provided the distribution of the number of uncovered gaps on the circle in this context. Following their work, T. Huillet obtained the joint distribution of the lengths of the connected components [65]. In [119], A. F. Siegel conjectured that $p_{\text {cov }}(n, \mu)$ satisfies a monotonicity result which is proved in [22] and is the following: if two probability distributions $\mu, v$ on $(0,1)$ are such that $\mu \leq v$ for the convex order, see e.g. [93, Chapter 1], then $p_{\text {cov }}(n, \mu) \leq p_{\text {cov }}(n, \mu)$. In particular, thanks to Jensen's inequality, this implies that $p_{\text {cov }}(n, x) \leq p_{\text {cov }}(n, \mu)$ where $x$ is the mean of $\mu$.

To the best of our knowledge, the most recent contribution in higher dimension is due to P. Bürgisser, F. Cucker and M. Lotz [21] and contains on one hand an exact formula for the probability to cover the sphere with $n$ spherical caps of fixed angular radius when this radius is larger than $\pi / 2$ and on the other hand an upper bound for this probability when the angular radius is less than $\pi / 2$.

Finally, a related question introduced by A. Dvoretzky in 1956 concerns the covering of the circle by an infinite number of intervals $\left(I_{n}\right)_{n}$ with deterministic lengths $\left(\ell_{n}\right)_{n}$ such that the sequence $\left(\ell_{n}\right)_{n}$ is non-increasing [39]. In 1972, L. A. Shepp showed that the circle is covered infinitely often with probability 1 if and only if the series $\sum_{n=1}^{\infty} n^{-2} \exp \left(\ell_{1}+\cdots+\ell_{n}\right)$ is divergent [118].

\subsection{A few basics on the Boolean model}

A natural extension of the bicycle wheel problem consists in considering random coverings of the Euclidean space by so-called grains with random positions and possibly random shapes or sizes. Considering the discussion on the translation invariance in Section 2, we construct directly such a model in $\mathbb{R}^{d}$. Let $\mathscr{P}_{\lambda}$ be a homogeneous Poisson point process of intensity $\lambda$ and let $K$ be a fixed non-empty compact set of $\mathbb{R}^{d}$, called the grain. Then the associated Boolean model is defined as the random set $\bigcup_{x \in \mathscr{P}_{\lambda}}(x+K)$, sometimes also called the occupied phase of the Boolean model. In the case when $K=B_{r}(o), r>0$, it was introduced by E. N. Gilbert in 1961 as a simplified approximation of the coverage of a radio transmission network where each individual can send a signal up to distance $r$ [43]. When $K$ is a random grain, for instance $K=B_{R}(o)$ with $R$ a non-negative random variable, the model can be extended in the following way: the occupied phase is $\bigcup_{x \in \mathscr{P}_{\lambda}}\left(x+K_{x}\right)$ where $\left(K_{x}\right)_{x}$ is a collection of i.i.d. copies of $K$ and independent of the Poisson point process $\mathscr{P}_{\lambda}$.

The Boolean model is well-adapted in a series of applied situations including flow in porous media [66], conduction in dispersions [78] and the elastic behavior of composites [133]. In practice, there are of course lots of fundamental statistical 


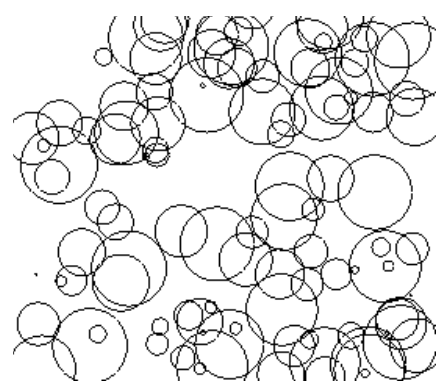

Fig. 10 Simulation of the Boolean model in the unit square in the case $\lambda=100$ and $K=B_{R}(o)$ where $R$ is uniform on $(0,0.1)$.

issues related in particular to the estimation of the intensity or of the grain distribution from the observation of the intersection of the Boolean model with a window or from sections or projections of this intersection on lower-dimensional subspaces. We shall omit this aspect and describe only the following probabilistic questions related to the model:

(a) estimating the covering probability of a particular set;

(b) concentrating on percolation, i.e. looking for the existence of an unbounded connected component of either the occupied or vacant phase;

(c) studying the geometry of the occupied or vacant phase or of their connected components.

Problem (a). In the eighties, L. Flatto and D. J. Newman followed by S. Janson investigated the distribution of the number of random balls with fixed radius necessary to cover a bounded subset of $\mathbb{R}^{d}$ or a Riemannian manifold in two seminal works $[41,67]$. Though this distribution is not explicit, S. Janson showed in particular a convergence in distribution, when the radius goes to 0 , of the renormalized number to a Gumbel law. Regarding the covering of the whole space $\mathbb{R}^{d}$, it is shown in [53, Theorem 3.1] and [83, Proposition 7.3] that $\mathbb{R}^{d}=\bigcup_{x \in \mathscr{P}_{\lambda}} B_{R}(x)$ occurs almost surely when $R^{d}$ is a non-integrable random variable and if not, the vacant set has infinite Lebesgue measure almost surely. P. Hall obtained upper and lower bounds for the probability of not covering $\mathbb{R}^{d}$ when $R$ is deterministic, see [53, Theorem 3.11]. These results have been recently extended by a study of the covering of $\mathbb{R}^{d}$ by unions of balls $B_{r}(x)$ when the couples $(x, r)$ belong to a Poisson point process in $\mathbb{R}^{d} \times(0, \infty)[14]$.

Problem (b) This question has been treated mostly in the case of i.i.d. grains of type $B_{R}(o)$ where $R$ is a non-negative random variable. In [52], P. Hall shows notably that if $E\left(R^{2 d-1}\right)$ is finite, then there exists a critical intensity $\lambda_{c} \in(0, \infty)$ such that if $\lambda<\lambda_{c}$, all the connected components of the occupied phase are bounded almost surely. A breakthrough due to J.-B. Gouéré [47] extends P. Hall's result in the 
following way: there exists a positive critical intensity $\lambda_{c}$ under which all connected components of the occupied phase are bounded if and only if $E\left(R^{d}\right)$ is finite. We also refer to [48] for the extension of that result for unions of balls $B_{r}(x)$ when the couples $(x, r)$ belong to a Poisson point process in $\mathbb{R}^{d} \times(0, \infty)$.

In the reference book [83] by R. Meester and R. Roy, it is shown that for any such Poisson Boolean model, the number of unbounded connected components of the occupied (resp. vacant) phase is either 0 or 1 almost surely, see Theorems 3.6 and 4.6 therein. Moreover, in the particular case of a two-dimensional Boolean model with almost surely bounded radii, criticality of the occupied and vacant phases coincide, i.e. there exists $\lambda_{c} \in(0, \infty)$ such that for $\lambda<\lambda_{c}$, there is possible percolation of the vacant phase and no unbounded component of the occupied phase almost surely, for $\lambda=\lambda_{c}$, neither the occupied phase nor the vacant phase percolates and for $\lambda>\lambda_{c}$, there is possible percolation of the occupied phase and no unbounded component of the vacant phase, see [83, Theorems 4.4, 4.5]. The equality of the two critical intensities of the vacant and occupied phases has been proved without the condition of almost surely bounded radii in two very recent works due to M. Penrose [99] and to D. Ahlberg, V. Tassion, and A. Teixeira [1].

Problem (c). The first formulas which connect the mean values of the characteristics of the grain to the mean values of the characteristics of the Boolean model intersected with a window are available in seminal papers due to R. E. Miles [89] and P. Davy [30]. A few decades later, [54] investigates large deviation probabilities for the occupied volume in a window. More recently, in [61], asymptotic covariance formulas and central limit theorems are derived for a large set of geometric functionals, including intrinsic volumes and so-called Minkowski tensors. We describe below two very simple examples of questions related to (c).

\subsubsection{The spherical contact distribution of the vacant phase}

We aim at determining the so-called spherical contact distribution of the vacant phase, i.e. the distribution of the radius $R_{c}$ of the largest ball centered at $o$ and included in the vacant phase, conditional on the event $\left\{o \notin \bigcup_{x \in \mathscr{P}_{\lambda}}(x+K)\right\}$. We observe that $R_{c} \geq r$ means that there is no point of $\mathscr{P}_{\lambda}$ at distance less than $r$ from $-K$. Consequently, we get

$$
\begin{aligned}
P(R \geq r) & =P\left(\mathscr{P}_{\lambda} \cap\left(-K+B_{r}(o)\right)=\emptyset\right) \\
& =\exp \left(-\lambda V_{d}\left(K+B_{r}(o)\right)\right) .
\end{aligned}
$$

When $K$ is a convex body, we can use the Steiner formula (4) and get that

$$
P(R \geq r)=\exp \left(-\lambda \sum_{k=0}^{d} \kappa_{d-k} V_{k}(K) r^{d-k}\right) .
$$


This calculation shows that when the grain $K$ is convex, the quantity $\log (P(R \geq r))$ is a polynomial in $r$. In [62], it is shown that the converse is not true in general, unless the spherical contact distribution is replaced by another contact distribution.

\subsubsection{The number of grains in a typical connected component of the occupied phase}

Similarly to the construction in Section 2 of the typical cell of a stationary tessellation, there is a way to define a typical connected component of the occupied phase: we add a deterministic point at the origin to the homogeneous Poisson point process and consider the connected component containing the origin of $\bigcup_{x \in \mathscr{P}_{\lambda} \cup\{o\}}(x+K)$. The aim of the calculation below is to derive a general formula for the distribution of the number $\mathscr{N}_{o}$ of grains contained in that connected component. The method below follows the work by M. Penrose [97], see also [102]. For any $n \geq 0$, we get

$$
P\left(\mathscr{N}_{o}=n+1\right)=E\left(\sum_{\left\{x_{1}, \cdots, x_{n}\right\} \in \mathscr{P}_{\lambda}^{(n)}} F\left(\left\{x_{1}, \cdots, x_{n}\right\}, \mathscr{P}_{\lambda}\right)\right)
$$

where $\mathscr{P}_{\lambda}^{(n)}$ is the set of finite subsets of $\mathscr{P}_{\lambda}$ with exactly $n$ elements and the functional $F\left(\left\{x_{1}, \cdots, x_{n}\right\}, \mathscr{P}_{\lambda}\right)$ is the indicator function of the event that the union $(o+K) \cup\left(x_{1}+K\right) \cup \cdots \cup\left(x_{n}+K\right)$ is connected and all the remaining grains $(x+K)$, $x \in \mathscr{P}_{\lambda} \backslash\left\{x_{1}, \cdots, x_{n}\right\}$ are disconnected from that union. The expectation in (38) can be made explicit thanks to Mecke's formula for Poisson point processes, see e.g. [114, Corollary 3.2.3]. We get indeed, for $n \geq 1$,

$$
\begin{aligned}
P\left(\mathscr{N}_{o}=n+1\right)= & \frac{\lambda^{n}}{n !} \int E\left(F\left(\left\{x_{1}, \cdots, x_{n}\right\}, \mathscr{P}_{\lambda} \cup\left\{x_{1}, \cdots, x_{n}\right\}\right) \mathrm{d} x_{1} \cdots \mathrm{d} x_{n}\right. \\
= & \frac{\lambda^{n}}{n !} \int \mathbf{1}_{\left\{\bigcup_{0 \leq k \leq n}\left(x_{i}+K\right) \text { connected }\right\}} \\
& P\left(\forall x \in \mathscr{P}_{\lambda},(x+K) \cap \bigcup_{0 \leq k \leq n}\left(x_{i}+K\right)=\emptyset\right) \mathrm{d} x_{1} \cdots \mathrm{d} x_{n}
\end{aligned}
$$

where for sake of simplicity, the origin $o$ has been denoted by $x_{0}$. Using again the fact that $\mathscr{P}_{\lambda}$ is a Poisson point process, we deduce that

$$
P\left(\mathscr{N}_{o}=n+1\right)=\frac{\lambda^{n}}{n !} \int \mathbf{1}_{\left\{\bigcup_{0 \leq k \leq n}\left(x_{i}+K\right) \text { connected }\right\}} e^{-\lambda V_{d}\left(\left(\bigcup_{0 \leq k \leq n}\left(x_{i}+K\right)\right)+(-K)\right)} \mathrm{d} x_{1} \cdots \mathrm{d} x_{n} .
$$

When $K=B_{r}(o)$ for fixed $r>0$, the previous formula becomes

$$
P\left(\mathscr{N}_{o}=n+1\right)=\frac{\lambda^{n}}{n !} \int \mathbf{1}_{\left\{\bigcup_{0 \leq k \leq n} B_{r}\left(x_{i}\right) \text { connected }\right\}} e^{-\lambda V_{d}\left(\bigcup_{0 \leq k \leq n} B_{2 r}\left(x_{i}\right)\right)} \mathrm{d} x_{1} \cdots \mathrm{d} x_{n} .
$$

K. Alexander [2] showed that when $\lambda \rightarrow \infty$, this probability satisfies 


$$
\log P\left(\mathscr{N}_{o}=n+1\right)=-\lambda \kappa_{d} r^{d}+(d-1) n \log \left(\frac{\lambda}{n}\right)+O(1)
$$

where $f(\lambda)=O(g(\lambda))$ means that the function $f / g$ is bounded for large $\lambda$. A side result is the so-called phenomenon of compression which says roughly that in a high-density Boolean model, the density inside a bounded connected component is larger than the ambient density.

Acknowledgements. The author warmly thanks two anonymous referees for their careful reading of the original manuscript, resulting in an improved and more accurate exposition.

\section{References}

1. Ahlberg, D., Tassion, V., Teixeira, A.: Existence of an unbounded vacant set for subcritical continuum percolation. Available via Arxiv. https://arxiv.org/abs/1706.03053 (2017)

2. Alexander, K. S.: Finite clusters in high-density continuous percolation: compression and sphericality. Probab. Theory Related Fields 97, 35-63 (1993)

3. Ambartzumian, R. V.: A synopsis of combinatorial integral geometry. Adv. Math. 37, 1-15 (1980)

4. Avram, F., Bertsimas, D.: On central limit theorems in geometrical probability. Ann. Appl. Probab. 3 4, 1033-1046 (1993)

5. Baccelli, F., Zuyev, S.: Poisson-Voronoi Spanning Trees with Applications to the Optimization of Communication Networks. Operations Research 47, 619-631 (1999)

6. Barbier, J.-E.: Note sur Ie probleme de l'aiguille et Ie jeu du joint couvert. J. Mathématiques Pures et Appliquées 5, 273-286 (1860)

7. Bárány, I.: Random polytopes in smooth convex bodies. Mathematika 39, 81-92 (1992)

8. Bárány, I.: Sylvesters question: the probability that $\mathrm{n}$ points are in convex position. Ann. Probab. 27, 2020-2034 (1999)

9. Bárány, I.: A note on Sylvesters four-point problem. Studia Sci. Math. Hungar. 38, 733-77 (2001)

10. Baryshnikov, Y.M., Vitale, R.A.: Regular simplices and Gaussian samples. Discrete Comput. Geom. 11, 141-147 (1994)

11. Baumstark, V., Last, G.: Gamma distributions for stationary Poisson flat processes. Adv. in Appl. Probab. 41, 911-939 (2009)

12. Bertrand, J.: Calcul des probabilités. Gauthier-Villars, Paris (1889)

13. Biehl, T.: Über Affine Geometrie XXXVIII, Über die Schüttlung von Eikörpern. Abh. math. Semin. Hamburg Univ. 2, 69-70 (1923)

14. Biermé, H., Estrade, A.: Covering the whole space with Poisson random balls. ALEA Lat. Am. J. Probab. Math. Stat. 9, 213-229 (2012)

15. Blaschke, W.: Lösung des "Vierpunktproblems" von Sylvester aus der Theorie der geometrischen Wahrscheinlichkeiten. Leipziger Berichte 69, 436-453 (1917)

16. Blaschke, W.: Vorlesungen über Differentialgeometrie II: Affine Differentialgeometrie. Springer-Verlag, Berlin (1923)

17. Blaschke, W.: Integralgeometrie 2: Zu Ergebnissen von M.W. Crofton. Bull. Math. Soc. Roum. Sci. 37, 3-11 (1935)

18. Bosq, D., Caristi, G., Deheuvels, P., Duma A., Gruber, P., Lo Bosco, D., Pipitone, V.: Marius Stoka: Ricerca Scientifica dal 1951 al 2013, Vol. III. Edizioni SGB, Messina (2014)

19. Buchta, C.: An identity relating moments of functionals of convex hulls. Discrete Comput. Geom. 33, 125-142 (2005) 
20. Buffon, G.-L. Leclerc, Comte de: Histoire naturelle, générale et particulière, avec la description du cabinet du Roy. Tome Quatrième. Imprimerie Royale, Paris (1777)

21. Bürgisser, P., Cucker, F., Lotz, M.: Coverage processes on spheres and condition numbers for linear programming. Ann. Probab. 38, 570-604 (2010)

22. Calka P.: The distributions of the smallest disks conta ining the Poisson-Voronoi typical cell and the Crofton cell in the plane Adv. in Appl. Probab. 34, 702-717 (2002)

23. Calka, P.: Tessellations. In: Kendall, W.S., Molchanov, I. (eds.) New perspectives in stochastic geometry, pp. 145-169. Oxford University Press, Oxford (2010)

24. Calka, P.: Asymptotic methods for random tessellations. In: Spodarev, E. (ed.) Stochastic geometry, spatial statistics and random fields. Lecture Notes in Math. 2068, 183-204, Springer, Heidelberg (2013)

25. Calka, P., Schreiber, T., Yukich, J. E.: Brownian limits, local limits and variance asymptotics for convex hulls in the ball. Ann. Probab. 41, 50-108 (2013)

26. Carathéodory, C., Study, E.: Zwei Beweise des Satzes daß der Kreis unter allen Figuren gleichen Umfanges den größten Inhalt hat. Math. Ann. 68, 133-140 (1910)

27. Carnal, H.: Die konvexe Hülle von $n$ rotationssymmetrisch verteilten Punkten. Z. Wahrscheinlichkeit. und verw. Gebiete 15, 168-176 (1970)

28. Cauchy, A. : Notes sur divers théorèmes relatifs à la rectification des courbes, et à la quadrature des surfaces. C. R. Acad. Sci. Paris 13, 1060-1063 (1841)

29. Chenavier, N.: A general study of extremes of stationary tessellations with examples. Stochastic Process. Appl. 124, 2917-2953 (2014)

30. Davy, P.: Projected thick sections through multi-dimensional particle aggregates. J. Appl. Probab. 13, 714-722 (1976)

31. Chiu, S. N., Stoyan, D., Kendall, W. S., Mecke, J.: Stochastic geometry and its applications. Third edition. Wiley Series in Probability and Statistics. John Wiley \& Sons, Ltd., Chichester (2013)

32. Coupier, D., Dereudre, D.: Continuum percolation for quermass interaction model. Electron. J. Probab. 19(35), 19 pp. (2014)

33. Cowan, R.: The use of ergodic theorems in random geometry. Adv. in Appl. Probab. 10, 47-57 (1978)

34. Crofton, M. W.: On the Theory of Local Probability, Applied to Straight Lines Drawn at Random in a Plane; The Methods Used Being Also Extended to the Proof of Certain New Theorems in the Integral Calculus. Philos. Trans. of the Roy. Soc. of London. 156, 181-199 (1868)

35. Daley, D. J.: Asymptotic properties of stationary point processes with generalized clusters. Z. Wahrscheinlichkeitstheorie und Verw. Gebiete 21, 65-76 (1972)

36. Daley, D. J., Vere-Jones, D.: An introduction to the theory of point processes. Springer Series in Statistics. Springer-Verlag, New York (1988)

37. Descartes, R.: Principia philosophiae, Louis Elzevir, Amsterdam (1644)

38. Domb, C.: Covering by random intervals and one-dimensional continuum percolation. J. Stat. Phys. 55, (1989)

39. Dvoretzky, A.: On covering a circle by randomly placed arcs. Proc. Nat. Acad. Sci. U.S.A. 42, 199-203 (1956)

40. Efron, B.: The convex hull of a random set of points. Biometrika 52, 331-343 (1965)

41. Flatto, L., Newman D. J.: Random coverings. Acta Math. 138, 241-264 (1977)

42. Franken, P., König, D., Arndt, U., Schmidt, V.: Queues and point processes. AkademieVerlag, Berlin (1981)

43. Gilbert, E. N.: Random plane networks. J. Soc. Indust. Appl. Math. 9, 533-543 (1961)

44. Goldman, A.: Sur une conjecture de D. G. Kendall concernant la cellule de Crofton du plan et sur sa contrepartie brownienne. Ann. Probab. 26, 1727-1750 (1998)

45. Goldman, A.: The Palm measure and the Voronoi tessellation for the Ginibre process. Ann. Appl. Probab. 20, 90-128 (2010)

46. Goudsmit, S.: Random distribution of lines in a plane. Rev. Mod. Phys. 17, 321-322 (1945)

47. Gouéré, J.-B.: Subcritical regimes in the Poisson Boolean model of continuum percolation. Ann. Probab. 36, 1209-1220 (2008) 
48. Gouéré, J.-B.: Subcritical regimes in some models of continuum percolation. Ann. Appl. Probab. 19, 1292-1318 (2009)

49. Gouéré, J.-B., Marchand, R.: Nonoptimality of constant radii in high dimensional continuum percolation. Ann. Probab. 44, 307-323 (2016)

50. Groemer, H.: On some mean values associated with a randomly selected simplex in a convex set. Pacific J. Math. 45, 525-533 (1973)

51. Hadwiger, H.: Vorlesungen ber Inhalt, Oberfläche und Isoperimetrie. Springer-Verlag, Berlin (1957)

52. Hall, P.: On continuum percolation. Ann. Probab. 13, 1250-1266 (1985)

53. Hall, P.: Introduction to the Theory of Coverage Processes. Wiley, New York (1988)

54. Heinrich, L.: Large deviations of the empirical volume fraction for stationary Poisson grain models. Ann. Appl. Probab. 15, 392-420 (2005)

55. Heinrich, L., Muche, L.: Second-order properties of the point process of nodes in a stationary Voronoi tessellation. Math. Nachr. 281, 350-375 (2008), Erratum Math. Nachr. 283, 1674$1676(2010)$

56. Heinrich, L., Schmidt, H., Schmidt, V.: Limit theorems for stationary tessellations with random inner cell structures. Adv. in Appl. Probab. 37, 25-47 (2005)

57. Heinrich, L., Schmidt, H., Schmidt, V.: Central limit theorems for Poisson hyperplane tessellations. Ann. Appl. Probab. 16, 919-950 (2006)

58. Hilhorst, H. J.: Asymptotic statistics of the n-sided planar Poisson-Voronoi cell. I. Exact results. J. Stat. Mech. Theory Exp. 9, P09005 (2005)

59. Hörrmann, J., Hug, D., Reitzner, M., Thle, C.: Poisson polyhedra in high dimensions. Adv. Math. 281, 1-39 (2015)

60. Hug, D.: Random polytopes. In: Spodarev, E. (ed.) Stochastic geometry, spatial statistics and random fields. Lecture Notes in Math. 2068, 205-238, Springer, Heidelberg (2013)

61. Hug, D., Last, G., Schulte, M.: Second order properties and central limit theorems for geometric functionals of Boolean models. Ann. Appl. Probab. 26, 73-135 (2016)

62. Hug, D., Last, G., Weil, W.: Polynomial parallel volume, convexity and contact distributions of random sets. Probab. Theory Related Fields 135, 169-200 (2006)

63. Hug, D., Reitzner, M., Schneider, R.: The limit shape of the zero cell in a stationary Poisson hyperplane tessellation. Ann. Probab. 32, 1140-1167 (2004)

64. Hug, D., Schneider, R.: Asymptotic shapes of large cells in random tessellations. Geom. Funct. Anal. 17, 156-191 (2007)

65. Huiller, T.: Random covering of the circle: the size of the connected components. Adv. in Appl. Probab. 35, 563-582 (2003)

66. Hunt, A., Ewing, R., Ghanbarian, B.: Percolation theory for flow in porous media. Third edition. Lecture Notes in Physics 880, Springer, Cham (2014)

67. Janson, S.: Random coverings in several dimensions. Acta Math. 156, 83-118 (1986)

68. Jaynes, E. T.: The well-posed problem. Foundations of Physics 3, 477-492 (1973)

69. Kendall, D. G.: Foundations of a theory of random sets. Stochastic geometry (a tribute to the memory of Rollo Davidson), pp. 322-376, Wiley, London (1974)

70. Kendall, M.G., Moran, P.A.P.: Geometrical Probability. Charles Griffin, London (1963)

71. Kingman, J. F. C.: Random secants of a convex body. J. Appl. Probab. 6, 660-672 (1969)

72. Kingman, J. F. C.: Poisson Processes. Clarendon Press, Oxford (1993)

73. Klain, D. A., Rota, G.-C.: Introduction to geometric probability. Cambridge University Press, Cambridge (1997)

74. Kolmogorov, A. N.: Grundbegriffe der Wahrscheinlichkeitsrechnung. Springer-Verlag, Berlin (1933)

75. Last, G., Penrose, M.: Lectures of the Poisson process. Cambridge University Press, Cambridge (2017)

76. Lefebvre, W., Philippe, T., Vurpillot, F.: Application of Delaunay tessellation for the characterization of solute-rich clusters in atom probe tomography. Ultramicroscopy 111, 200-206 (2011)

77. Marckert, J.-F.: The probability that $\mathrm{n}$ random points in a disk are in convex position. To appear in Braz. J. of Probab. and Stat. (2017) 
78. Markov, K. Z., Christov, C. I.: On the problem of heat conduction for random dispersions of spheres allowed to overlap. Mathematical Models and Methods in Applied Sciences 2, 249-269 (1992)

79. Matérn, B.: Spatial variation: Stochastic models and their application to some problems in forest surveys and other sampling investigations. Meddelanden Fran Statens Skogsforskningsinstitut 49, Stockholm (1960)

80. Matheron, G.: Random sets and integral geometry. Wiley Series in Probability and Mathematical Statistics. John Wiley \& Sons, New York-London-Sydney (1975)

81. Mecke, J.: Stationäre zufällige Masse auf lokalkompakten Abelschen Gruppen. Z. Wahrscheinlichkeitstheorie und Verw. Gebiete 9, 36-58 (1967)

82. Mecke, J.: On the relationship between the 0-cell and the typical cell of a stationary random tessellation. Pattern Recognition 32, 16451648 (1999)

83. Meesters, R., Roy, R.: Continuum Percolation. Cambridge University Press, New York (1996)

84. Meijering, J. L.: Interface area, edge length and number of vertices in crystal aggregates with random nucleation. Philips Res. Rep. 8, 270-90 (1953)

85. Miles, R. E.: Random polygons determined by random lines in a plane I. Proc. Natl. Acad. Sci. USA 52, 901-907 (1964)

86. Miles, R. E.: Random polygons determined by random lines in a plane II. Proc. Natl. Acad. Sci. USA 52, 1157-1160 (1964)

87. Miles, R. E.: The random division of space. Suppl. Adv. in Appl. Probab., 243-266 (1972)

88. Miles, R. E.: The various aggregates of random polygons determined by random lines in a plane. Adv. Math. 10, 256-290 (1973)

89. Miles, R. E.: Estimating aggregate and overall characteristics from thich sections by transmission microscopy. J. Microsc. 107, 227-233 (1976)

90. Møller, J.: Random tessellations in $\mathbb{R}^{d}$. Adv. in Appl. Probab. 21, 37-73 (1989)

91. Møller, J.: Random JohnsonMehl tessellations. Adv. in Appl. Probab. 24, 814-844 (1992)

92. Møller, J.: Lectures on Random Voronoi Tessellations. Lecture Notes in Statist. 87, Springer, New York (1994)

93. Müller, A., Stoyan, D.: Comparison methods for stochastic models and risks. Wiley Series in Probability and Statistics. John Wiley \& Sons Ltd., Chichester (2002)

94. Nagel, W., Weiss, V.: Crack STIT tessellations: characterization of stationary random tessellations stable with respect to iteration. Adv. in Appl. Probab. 37, 859-883 (2005)

95. Neveu, J.: Processus ponctuels. In: École d'été de Probabilités de Saint-Flour, pp. 249-445. Lecture Notes in Mathematics 598, Springer-Verlag, Berlin (1977)

96. Palm, C.: Intensitätsschwankungen im Fernsprechverkehr. Ericsson Technics 44, 1-189 (1943)

97. Penrose, M.: On a continuum percolation model. Adv. in Appl. Probab. 23, 536-556 (1991)

98. Penrose, M.: Random Geometric Graphs. Oxford University Press, Oxford (2003)

99. Penrose, M. : Non-triviality of the vacancy phase transition for the Boolean model. Available via Arxiv. https://arxiv.org/abs/1706.02197 (2017)

100. Pfiefer, R. E.: The historical development of J. J. Sylvester's four point problem. Math. Magazine 62, 309-317 (1989)

101. Poincaré, H.: Calcul des probabilités. Gauthier-Villars, Paris (1912)

102. Quintanilla, J., Torquato, S.: Clustering in a continuum percolation model. Adv. in Appl. Probab. 29, 327-336 (1997)

103. Redenbach, C.: On the dilated facets of a Poisson-Voronoi tessellation. Image Anal. Stereol. 30, 31-38 (2011)

104. Reitzner, M.: Stochastical approximation of smooth convex bodies. Mathematika 51, 11-29 (2004)

105. Reitzner, M.: Central limit theorems for random polytopes. Probab. Theory Relat. Fields 133, $483-507(2005 \mathrm{a})$

106. Reitzner, M.: The combinatorial structure of random polytopes. Adv. Math. 191, 178-208 (2005)

107. Reitzner, M.: Random polytopes. In: Kendall, W.S., Molchanov, I. (eds.) New perspectives in stochastic geometry, pp. 45-76. Oxford University Press, Oxford (2010) 
108. Rényi, A., Sulanke, R.: Über die konvexe Hülle von n zufällig gewählten Punkten. Z. Wahrscheinlichkeitsth. verw. Geb. 2, 75-84 (1963)

109. Rényi, A., Sulanke, R.: Über die konvexe Hülle von n zufällig gewählten Punkten. II. Z. Wahrscheinlichkeitsth. verw. Geb. 3, 138-147 (1964)

110. Schneider, R.: Convex Bodies: the Brunn-Minkowski Theory. Cambridge University Press, Cambridge (1993)

111. Santaló, L. A.: Integral geometry and geometric probability. Encyclopedia of Mathematics and its Applications, Vol. 1. Addison-Wesley Publishing Co., Reading, Mass.-LondonAmsterdam (1976)

112. Schneider, R.: Random hyperplanes meeting a convex body. Z. Wahrsch. Verw. Gebiete 61, 379-387 (1982)

113. Schneider, R.: Integral geometric tools for stochastic geometry. In: Weil, W. (eds.) Stochastic geometry. Lectures given at the C.I.M.E. Summer School held in Martina Franca, pp. 119184. Lecture Notes in Mathematics 1892, Springer-Verlag, Berlin (2007)

114. Schneider, R., Weil, W.: Stochastic and integral geometry. Springer-Verlag, Berlin (2008)

115. Schneider, R., Weil, W.: Classical stochastic geometry. In: Kendall, W.S., Molchanov, I. (eds.) New perspectives in stochastic geometry, pp. 1-42. Oxford University Press, Oxford (2010)

116. Schneider, R., Wieacker, J. A.: Random polytopes in a convex body. Z. Wahrsch. Verw. Gebiete 52, 69-73 (1980)

117. Serra, J.: Image analysis and mathematical morphology. Academic Press Inc., London (1984)

118. Shepp, L. A.: Covering the circle with random arcs. Israel J. of Math. 11, 328-345 (1972)

119. Siegel, A. F.: Random space filling and moments of coverage in geometrical probability. J. Appl. Probab. 15, 340-355 (1978)

120. Siegel, A. F., Holst, L.: Covering the circle with random arcs of random sizes. J. Appl. Probab. 19, 373-381 (1982)

121. Solomon, H.: Geometric probability. CBMS-NSF Regional Conference Series in Applied Mathematics 28, SIAM, Philadelphia (1978)

122. Steiner, J.: Über parallele Flächen. Monatsber. Preuss. Akad. Wiss., 114-118, Berlin (1840)

123. Stevens, W. L.: Solution to a geometrical problem in probability. Ann. Eugenics 9, 315-320 (1939)

124. Stoyan, D.: Applied stochastic geometry: a survey. Biometrical J. 21), 693-715 (1979)

125. Sylvester, J. J.: Problem 1491. The Educational Times, London, April (1864)

126. Valtr, P.: Probability that $\mathrm{n}$ random points are in convex position. Discr. \& Comput. Geom. 13,637-643 (1995)

127. Valtr, P.: The probability that $\mathrm{n}$ random points in a triangle are in convex position. Combinatorica 16, 567-573 (1996)

128. Vu, V. H.: Sharp concentration of random polytopes. Geom. Funct. Anal. 15, 1284-1318 (2005)

129. Weil, W.: Point processes of cylinders, particles and flats. Acta Appl. Math. 9, 103-136 (1987)

130. Weiss, V., Cowan, R.: Topological relationships in spatial tessellations. Adv. in Appl. Probab. 43, 963-984 (2011)

131. Wendel, J.G.: A problem in geometric probability. Math. Scand. 11, 109-111 (1962)

132. Whitworth, W. A.: Choice and Chance. D. Bell and co., Cambridge (1870)

133. Willot, F., Jeulin, D.: Elastic behavior of composites containing Boolean random sets of inhomogeneities. Internat. J. Engrg. Sci. 47, 313-324 (2009)

134. New advances in geostatistics. Papers from Session Three of the 1987 MGUS Conference held in Redwood City, California, April 1315, 1987. Math. Geol. 20, pp. 285-475, Kluwer Academic/Plenum Publishers, Dordrecht (1988)

135. New perspectives in stochastic geometry. Edited by W. S. Kendall \& I. Molchanov. Oxford University Press, Oxford (2010) 